\title{
Best practices and tools for reporting reproducible fluorescence microscopy methods
}

\author{
Paula Montero Llopis ${ }^{1 凶}$, Rebecca A. Senft ${ }^{2}$, Tim J. Ross-Elliott ${ }^{1}{ }^{1}$, Ryan Stephansky ${ }^{\prime}$, Daniel P. Keeley ${ }^{3}$, \\ Preman Koshar $\mathbb{B}^{3}$, Guillermo Marqués $\mathbb{1}^{4}$, Ya-Sheng Gao ${ }^{5}$, Benjamin R. Carlson ${ }^{5}$, Thomas Pengo $\mathbb{D}^{6}$, \\ Mark A. Sanders $\mathbb{1}^{4}$, Lisa A. Cameron $\mathbb{1}^{5}$ and Michelle S. Itano $\mathbb{1}^{3}$
}

\begin{abstract}
Although fluorescence microscopy is ubiquitous in biomedical research, microscopy methods reporting is inconsistent and perhaps undervalued. We emphasize the importance of appropriate microscopy methods reporting and seek to educate researchers about how microscopy metadata impact data interpretation. We provide comprehensive guidelines and resources to enable accurate reporting for the most common fluorescence light microscopy modalities. We aim to improve microscopy reporting, thus improving the quality, rigor and reproducibility of image-based science.
\end{abstract}

T he development of new technologies and tools in light microscopy has had an important role in making biomedical research more quantitative and interdisciplinary. This allows researchers to tackle more challenging scientific questions and obtain a more in-depth understanding of complex biological systems. However, with the rapid deployment of many new technologies, it is difficult for researchers to maintain in-depth knowledge of their capabilities and limitations. In addition, it is not always clear to users of advanced imaging techniques how specific limitations in hardware and software configurations for each modality might lead to errors that could affect scientific conclusions and reproducibility. Accordingly, the methods section of a publication requires complete and accurate information on the imaging conditions used to allow experimental replication. However, Marqués et al. ${ }^{1}$ recently showed that lack of detail in describing image acquisition is a widespread problem in biomedical publications, especially when considering that imaging is critical in biomedical research.

Many publications focus on guidelines to successfully perform a microscopy experiment, including sample preparation, method validation and appropriate controls to ensure reproducibility ${ }^{2-5}$. However, there are still few resources to guide and assist researchers in writing rigorous and reproducible microscopy methods. This Perspective focuses on filling this need by proposing minimal guidelines to ensure rigor and reproducibility in fluorescence light microscopy.

\section{Scope of the guidelines}

The scope of this Perspective is to emphasize the importance of appropriate microscopy methods reporting and help educate researchers about microscopy components and parameters that impact data and conclusions. We showcase examples to demonstrate and highlight errors that can arise from insufficient reporting and provide resources to assist researchers with this task: an educational poster (Extended Data Fig. 1), comprehensive checklists (Supplementary Exhibits 1-4) and a customizable checklist-generating tool, MicCheck (Supplementary Exhibit 5). These guidelines are in line with the tier-based system developed in the OME-4DN project for metadata collection to ensure consistency across the microscopy community ${ }^{6}$.
The checklists and MicCheck enumerate and describe in detail the essential and recommended metadata for the most common fluorescence light microscopy applications: widefield, laser scanning (confocal and multiphoton) and spinning disk confocal experiments, for live and fixed samples. The essential, or minimally required, metadata are critical for the correct interpretation of microscopy data and must be reported to ensure rigor and reproducibility. The recommended metadata represent best practices, particularly when using custom-built microscopes and nonstandard image acquisition strategies, but these may not substantially impact the conclusions that may be drawn. Finally, we include a list of resources and initiatives to improve image-based reproducibility and a list of definitions for many of the terms discussed in this Perspective (Supplementary Tables 1 and 2) that can serve as a reference for more novice microscopy users.

We intend these materials to be generally appropriate for many of the most common light microscopy configurations and applications $^{7}$ but encourage researchers to use their own discretion and apply the recommendations as appropriate for their specific application and imaging system.

\section{Guidelines on reporting instrument metadata}

Illumination, light collection and wavelength selection. Illumination is critical to assess (1) how efficiently a fluorophore will be excited, (2) the relative illumination intensity for each fluorophore, (3) how reproducible intensity measurements are over time, (4) the probability of excitation cross-talk and (5) the compatibility of excitation filters ${ }^{8}$. How the excitation and collection of light from fluorophores is achieved can vary widely. Thus, the choice of hardware used to illuminate the sample and to collect the fluorescence signal emitted from the fluorophores will have a profound effect on data interpretation and the ability to make meaningful comparisons between datasets (Box 1). Specifically, the light source and the excitation and emission wavelength bandwidth and hardware (for example, the excitation filter and dichromatic mirror) used are essential metadata. The power density (irradiance) at the sample impacts the excitation efficiency of fluorophores, the photobleaching rate and the cumulative amount of light that can be collected from the sample during acquisition. While irradiance

'MicRoN Core, Harvard Medical School, Boston, MA, USA. '2Department of Genetics, Blavatnik Institute, Harvard Medical School, Boston, MA, USA. ${ }^{3}$ Neuroscience Microscopy Core, University of North Carolina, Chapel Hill, NC, USA. ${ }^{4}$ University Imaging Centers and Department of Neuroscience, University of Minnesota, Minneapolis, MN, USA. ${ }^{5}$ Duke Light Microscopy Core Facility, Duke University, Durham, NC, USA. ${ }^{6}$ University of Minnesota Informatics Institute, University of Minnesota, Minneapolis, MN, USA. 『e-mail: paula_monterollopis@hms.harvard.edu 


\section{Box 1 | Irradiance and quantitative fluorescence}

Irradiance, or illumination power density $\left(\mathrm{W} \mathrm{cm}^{-2}\right)$ at the sample, determines the excitation efficiency of fluorophores and impacts the photobleaching rate and the cumulative amount of light that can be collected from the sample during acquisition. The total irradiance at the sample plane depends on the light source, the hardware to select excitation wavelengths, the objective lens and the modality ${ }^{53,54}$.

If quantitative fluorescence intensity measurements are required, a more stable light source is advisable. Laser-based illumination usually results in higher irradiance than other types of illumination, as the light is coherent and typically has higher illumination power intensity than with other light sources. How modulation of the laser power is achieved also impacts irradiance. For example, a directly modulated laser with $100-\mathrm{mW}$ power output at the optical fiber tip will have higher irradiance than the same power output in a laser modulated through an AOTF, as this tunable filter results in a loss of light of approximately $40-50 \%$ (P.M.L., unpublished observation). How focused the laser light is (objective numerical aperture) will also determine the irradiance at the sample. When comparing and choosing a laser power output configuration for an imaging system, it is important to note that different manufacturers report the laser output differently. Some report power output directly at the source, while others report it at the tip of the optical fiber just before light enters the confocal scanner or the microscope. The light power can be adjusted in the acquisition software (often with a label indicating 'laser power'), but this adjustment may not be linear. The power should not be interpreted to indicate an exact percentage of the laser power output because of loss of light across optical surfaces on the way to the sample.

\section{The importance of irradiance in reproducibility}

Irradiance varies substantially with microscopy modality. In the case of single-point scanning confocal microscopy, the laser beam is focused into a single spot (whose size depends on the objective lens used) and therefore corresponds to higher irradiance than with other modalities such as spinning disk microscopy, where the laser is collimated and illuminates the entire field of view ${ }^{53}$. Photobleaching, phototoxicity and fluorophore saturation (when most fluorophores in the sample are in the excited state and there are therefore no molecules to absorb new photons) are also greatly impacted by irradiance and are a source of variability and irreproducibility.

While irradiance influences fluorophore excitation efficiency and photobleaching, considering its impact on sample health is critical to obtaining reproducible and biologically meaningful results. Light can induce DNA damage and oxidation of cellular components ${ }^{4,25}$. In addition to these damaging effects, fluorophore photobleaching further affects sample health by generating free radicals and other reactive species ${ }^{55}$. Together, these aspects will negatively impact cellular function and health. The extent of light-induced damage depends on the amount of light that the sample is subjected to, sometimes referred to as 'light overhead' $\left(\right.$ ref. $\left.{ }^{56}\right)$. While breaks between consecutive periods of illumination may allow for partial recovery, the effects of photodamage are cumulative ${ }^{57,58}$. The effects of excess illumination before starting the image capture routine (for example, while navigating and focusing through the eyepiece of the microscope) or during a time-lapse experiment (excess illumination or illumination overhead) will vary between instruments and can contribute to variability in sample health and photobleaching, resulting in irreproducibility.

Every effort should be made to minimize fluorescence excitation exposure before the experiment is acquired and to limit it to the specific time of the image capture needed to collect data $^{26}$. Other sources of illumination overhead include the time period of shutter opening and closing controlled by acquisition software, asynchronization between camera acquisition and illumination, stage movement during a $z$-stack acquisition and even environmental light exposure during sample storage.

\section{The challenges of measuring irradiance}

Thus, irradiance is a critical aspect and needs to be as consistent as possible between imaging sessions, especially when measuring intensity or when conducting live-cell imaging.

Providing information on the irradiance used in a particular experiment would greatly improve reproducibility. However, providing an accurate irradiance measurement at the sample presents several challenges. (1) This requires tools that most researchers or laboratories may not have available, such as optical power meters. Some power meters include a sensor area that fits on the stage, replacing the sample, thus enabling light intensity measurements at specific wavelengths at the sample plane. (2) Illumination overhead can be difficult to account for or to measure from the metadata. (3) There is a lack of standardization in the protocols used to collect irradiance measurements, leading to irreproducibility. Working group 1 of Quality Assessment and Reproducibility for Instruments \& Images in Light Microscopy (QUAREP; Illumination Power) consists of an international team working on the development of standard procedures to measure and standardize irradiance measurements and will seek input and consensus from the global community ${ }^{48}$.

While irradiance measurements are challenging to perform properly, it is still advisable to routinely monitor light intensity in a particular instrument to evaluate potential sources of variability and imprecision.

To measure irradiance at the sample, the illuminated area needs to be measured. In widefield or spinning disk systems, this can be done by bleaching an area in a uniform sample and collecting an image with a lower-magnification lens to measure the bleached area. The sample can even be created using a fluorescent highlighter pen to mark the bottom of a sample carrier. In single-point scanning microscopes, the size of the laser spot can be measured using the reflection mode and a spot scan.

We encourage researchers to connect with core facilities or other imaging scientists at their institution for assistance, as these groups often have the tools and protocols to assist in measurement and monitoring of illumination intensity. is critical for reproducibility and ideally should be carefully documented, it is challenging to measure, requiring dedicated tools and protocols (Box 1).

The signal that can be collected is determined by the hardware used. Well-matched filters produce brighter fluorescence at shorter exposures or lower excitation light intensities and improve overall detection, signal-to-noise ratio (SNR) and downstream data analysis. Under otherwise identical imaging conditions, the SNR of the fluorescent foci and membrane localization of the fusion protein in Fig. 1a are below the detection threshold using an emission filter that is not well matched to the fluorophore. This will lead to conflicting results if not well documented. Additionally, the sample requires higher illumination intensity to excite and detect the appropriate fluorescence signal to determine the localization 
a

'Green' emission

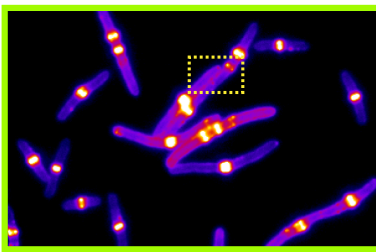

'Yellow' emission

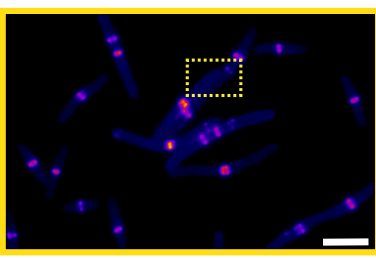

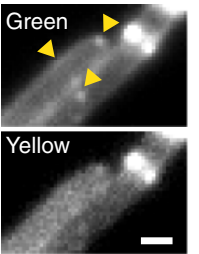

SYTO Deep Red

b

Mito-RFP
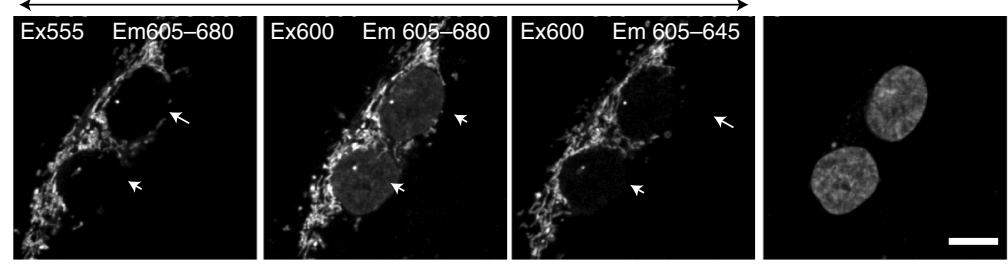

DAPI: 415-475 nm

DAPI: $415-595 \mathrm{~nm}$
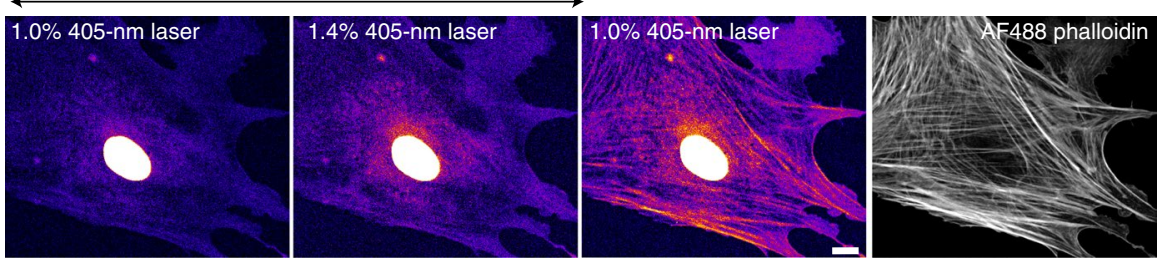
Same dichroic

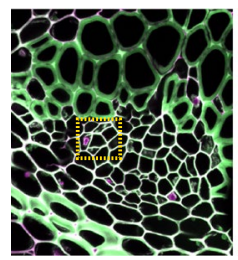
Different dichroic

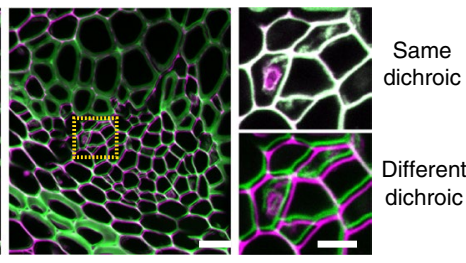

Fig. 1 | Illumination and wavelength selection impact signal detection, image quality and cross-talk between channels. a, Fluorescence images (using Fire LUT) of Escherichia coli cells expressing ZipA-sfGFP acquired under identical acquisition settings and excitation wavelengths with different emission filters. Left, the emission filter is well matched to sfGFP ('green'; Semrock FF01-515/30). Center, the emission filter is less well matched to the fluorescence filter ('yellow'; Semrock FF01-544/24). The yellow arrowheads indicate localization at the membrane and discrete foci. Right, violin plots of the fluorescence intensity of individual images. Encircled dots indicate the population mean ('green', $n=432$ cells; 'yellow', $n=432$ cells). Scale bars, $5 \mu \mathrm{m}$ and $1 \mu \mathrm{m}$ (magnification). b, Fluorescence images (using a grayscale LUT) of U2OS cells expressing Mito-RFP and labeled with SYTO Deep Red (nuclei) acquired with excitation at $555 \mathrm{~nm}$ (left) or $600 \mathrm{~nm}$ (center left) and emission collected from 605-680 nm. White arrowheads indicate SYTO Deep Red nuclear emission signal collected. Center right, same as in center left but with emission collection adjusted down to 605-645 nm, which almost completely removes the SYTO Deep Red cross-talk, indicated by white arrowheads. Right, SYTO Deep Red image. Scale bar, $10 \mu \mathrm{m}$. c, Fluorescence images (using a Fire LUT) of BPAE cells stained with DAPI and Alexa Fluor 488 phalloidin (right) imaged using a narrow-bandpass emission range of $415-475$ nm (left and center left) or a broad-bandpass emission range (center right). Center left, same as in left but with higher laser intensity. Scale bar, $10 \mu \mathrm{m}$. d, Two-color fluorescence imaging of Convallaria using a single multi-bandpass dichroic mirror for both channels (left) or a different dichroic mirror for each channel (center). Overlay images are pseudocolored green (488-nm excitation) and magenta (561-nm excitation). White indicates overlap between the two signals. Right, magnified views show the extent of xy shift between the two images using the same (top) or different (bottom) dichroic mirrors. Scale bars, $25 \mu \mathrm{m}$ and $5 \mu \mathrm{m}$ (magnification).

pattern. This can impact photobleaching and affect the health of the sample, which in turn could affect the localization pattern, leading to irreproducibility.

In multicolor imaging, in which the excitation and emission spectra of the different fluorophores may be partially overlapping, the appropriate selection and documentation of excitation and emission wavelengths (Supplementary Table 1) is critical to spectrally separate and quantify signals in different channels. In Fig. 1b, the localization pattern and intensity of the Mito-RFP signal differ depending on the excitation wavelength used. Using $600-\mathrm{nm}$ light to illuminate the sample results in an erroneous localization pattern and intensity levels, as they are partially due to cross-talk from a different fluorophore in the sample (nuclear SYTO Deep Red). This cross-talk can be minimized by illuminating the sample with $555-n m$ light. Figure 1c shows that using a narrow-bandpass emission range minimizes cross-talk between two channels (compare center right and left), even when the laser intensity is increased to improve SNR (center right). These examples highlight the need for appropriate controls to evaluate the extent of cross-talk between channels in multicolor experiments and how the lack of documentation of excitation and emission wavelengths may lead to irreproducibility.

Experiments in which multiple fluorophores need to be acquired simultaneously or with little temporal or spatial shift between 


\section{Box 2 | Method validation considerations}

Validating the methodology used in an experiment is critical for reproducibility, as it ensures that the measurements obtained reflect the biological process under study and are not artifacts due to the performance of the microscope. Thus, a careful assessment of the capabilities, limitations and performance of a particular microscope is essential to ensure rigor and reproducibility. Here we describe some selected procedures and measurements that should be considered when designing microscopy experiments. Supplementary Table 1 contains a list of selected reagents and resources to perform these tests, in addition to selected ongoing microscopy initiatives that are currently focusing on building resources.

Assessing microscope resolution. The point spread function (PSF) represents how a particular microscope affects light emitted from a single point. As light travels through the sample and the apertures within the microscope, including the objective, it diffracts, or spreads in space. The image of this point of light will consist of a central Gaussian spot surrounded by concentric diffraction rings of lower intensity. The PSF can be used to determine the resolving power of the system, in $x y$ and $z$, and how sample preparation can affect the performance of the microscope as well as to anticipate limitations in the experimental design. A sample of fluorescent beads with a diameter of approximately $100 \mathrm{~nm}$ can be used to collect a PSF image with a high-NA lens, as the beads serve as a point source of light. Of note, however, these beads tend to clump together, and, because they are diffraction limited, it may not be obvious during imaging that the collected PSF corresponds to more than one bead, resulting in an underestimate of the system's resolution. Thus, the stock solution needs to be vortexed and sonicated, and the brightest spots in the images should be avoided during PSF analysis. The full width at half-maximum intensity (FWHM) of the PSF in $x y$ and $z$ from individual beads provides a quantitative measurement for the resolution achievable ${ }^{16}$. PSFj is an excellent open-source tool to quantitatively evaluate many aspects of the PSF $^{59}$ (https://github. com/cmongis/psfj). Additionally, study 3 from the Association of Biomolecular Resource Facilities (ABRF) Light Microscopy Research Group (LMRG) focuses on producing reliable and reproducible protocols for measuring the PSF (https://www.abrf. org/light-microscopy-lmrg-).

Adjusting a correction collar. Variation in coverslip thickness or mismatch in refractive index in the sample results in spherical aberration that compromises axial resolution and signal intensity. Some lenses are designed with a correction collar to make fine adjustments to minimize spherical aberration. Correction collar adjustment can be tricky and cumbersome, especially in inverted microscopes, as access to the objective and correction collar is limited to the space between the nosepiece and the stage. New advancements in this arena include motorization and software control of the correction collar to allow for more precise and reproducible adjustments of the settings. Any bright structures within the sample can be used to adjust the correction collar. The ideal shape of a $\mathrm{PSF}_{z}$ is similar to an hourglass, with symmetrical diffraction patterns above and below the central focal plane. In the eyepiece, this would appear as concentric diffraction rings of similar size above and below the plane where a bead is in focus. In the case of spherical aberration, the shape of the $\mathrm{PSF}_{z}$ would no longer be symmetrical and the diffraction rings would only be apparent when defocusing in one direction. Slowly rotating the correction collar in either direction while examining the symmetry of the PSF with each change will help in adjusting the correction collar appropriately. Alternatively, the sharpness and overall intensity of an image can be examined before and after rotating the correction collar. The image will be sharpest and produce the brightest intensity when spherical aberration is minimized.

Assessing axial chromatic aberration. Chromatic aberration greatly impacts conclusions and measurements in 3D multicolor experiments. It can be due to the type of correction within the objective lens and/or to a mismatch in refractive index between the immersion medium and the sample mounting medium that results in different spherical aberration according to wavelength (for example, dispersion). Assessment of the extent of any axial shift between channels is essential to validate colocalization and distance measurements between objects, especially when their size is diffraction limited. TetraSpeck microspheres, which are stained with four different fluorescent dyes, gold beads, which are autofluorescent over a broad range of different wavelengths, or staining of the same target with two different fluorophores within a sample can be used to evaluate chromatic aberration. Ideally, these samples should be prepared in the same sample carrier, with the same coverslips and mounting/imaging media as for the sample and with the same image acquisition settings as in the multicolor experiment (for example, acquiring every wavelength in each focal plane). Image acquisition software may enable correction of chromatic aberration during acquisition if the mismatch in refractive index is known. Alternatively, this shift can be corrected after acquisition using different algorithms ${ }^{19}$.

Assessing $x y$ chromatic shift (channel registration). Registration or chromatic shift may be introduced by the optics, hardware selection (for example, multiple dichroic mirrors or filters not correctly set in the filter cube), relevant device precision (for example, stage movement), pinhole and collimator alignment, and the extent of instrument calibration ${ }^{11,55,60}$. Of note, chromatic shift introduced by the optics may not be consistent across the field of view and the location of the objects/sample to measure could introduce bias and irreproducibility in the measurements. TetraSpeck beads and other fiducial markers, such as gold beads and commercial calibration slides (for example, ArgoLight, GATTAquant or DNA-PAINT 80R nanoruler slides), are useful to assess and correct for possible chromatic shift. There are a number of both open-source and proprietary algorithms that enable alignment and correction of this shift. Importantly, the algorithms used to correct this shift may also introduce image warping aberrations and require careful validation using a known sample before application to a raw image.

Assessing cross-talk. Even when fluorophores and filter sets are carefully chosen, cross-talk remains one of the main concerns in both single- and multicolor imaging because it can result in erroneous interpretation of the results ${ }^{3,17,44,61}$. Unlabeled controls and singly labeled controls (in which the sample is labeled with each individual fluorophore or expresses each individual fluorescent protein) are used to determine the extent of excitation and emission cross-talk between channels. They serve to validate the selected hardware and acquisition settings to minimize cross-talk or can be used to calculate parameters to correct for any observed cross-talk.

Assessing and correcting evenness of illumination (flatfield). In most systems, illumination is not even across the field of view. This nonuniformity results in differences in fluorescence 


\section{Box 2 | Method validation considerations (continued)}

intensity that do not reflect the biology of the sample. Uneven illumination needs to be measured and, if necessary, corrected (shading or flatfield correction). Of note, errors can be introduced for subsequent measurements when applying a flatfield correction, so this processing step needs to be validated and evaluated. Slides containing high concentrations of quenchable fluorescent dyes such as fluorescein or Rose Bengal are ideal to determine the illumination pattern and collect a flatfield correction image ${ }^{62,63}$. The quenching properties of these dyes ensure that a thin and even section is illuminated. This is critical in widefield systems because differences in the thickness of a fluorescent slide will result in an apparent change in intensity that is not due to the illumination pattern and can therefore introduce errors when correcting the raw data. In other modalities, fluorescent plastic slides (for example, Chroma) can be used. These slides are very bright, so it is recommended to use an illumination wavelength that is not optimal to minimize the intensity (for example, use a Red slide to assess the illumination pattern of the green channel). In most single-point scanning confocal microscopes, illumination uniformity can be maximized by carefully adjusting the alignment of the collimator and pinhole. While such adjustments may not be accessible to most researchers, talking with vendors and imaging scientists can ensure that the system is properly aligned to minimize field nonuniformity.
Calibrating distances. The final pixel size in a digital image acquired on a digital camera depends on the size of the photodiode in the camera and the total magnification (objective, optovar and relay lens). The final pixel size in a digital image acquired with a PMT is based on the magnification of the objective, the area of the field of view scanned by the laser and the sampling interval of the PMT signal by the pixel clock. Careful calibration of the pixel size of the digital image can be achieved by imaging a micrometer ruler slide. A slide with a ruler with $0.01-\mathrm{mm}$-scale resolution etched into the glass can be used with different magnifications to calibrate distances ${ }^{64}$.

Photobleaching. Intensity measurements over time will be affected by photobleaching, even in the case of fixed samples, as the signal intensity will decrease over time as a result of fluorophore destruction and not the biology of the sample. The rate of photobleaching varies with the fluorophore used, illumination intensity and illumination mode. Minimizing photobleaching during the experiment is strongly advised, even for fixed samples. Correction can be applied by obtaining decay curves and correcting the decay function on fixed samples. Validation of this function is critical to avoid overcorrecting or introducing additional errors ${ }^{3,65,66}$ (ImageJ plugin CorrectBleach v2.0.2; https:// zenodo.org/record/30769\#.XIflKyhKg2w). channels (for example, ratiometric imaging and colocalization experiments) require the use and documentation of specific hardware to minimize chromatic shift (Box 2). This shift may be due to an improper angle of mounting for dichroic mirrors and imperfections on their thin-film interference coatings or to poor instrument calibration. Channel misregistration greatly impacts the conclusions from colocalization experiments and experiments aimed at quantifying distances between objects or intensity ratios between channels (Fig. 1d). Controls are critical in assessing the extent of chromatic shift and correcting it ${ }^{9-11}$ (Box 2 and Supplementary Table 1).

Objective lenses. Objective lenses are essential for image formation and impact all aspects of imaging; thus, they are one of the most critical components that need to be reported. Magnification is an important aspect of digital microscopy, as it contributes to the size of the field of view and, for camera-based microscopes, the pixel size (spatial sampling). Many researchers understand this, and in most cases this aspect is documented. However, objective lenses possess other characteristics that have an even greater impact on image formation and must be reported for others to reproduce an experiment.

Objective lenses contain spherical surfaces that focus the light into the sample and collect emitted fluorescence to form an image. These curved surfaces create different types of aberrations ${ }^{12-14}$. Manufacturers design objective lenses applying different aberration corrections depending on the intended application. These corrections are made assuming specific conditions such as coverslip thickness, refractive index of the medium used, temperature and illumination wavelengths. Some objective lenses are designed with correction collars, which allow for fine-tuning of the performance of the objective lens to compensate for sample-induced aberrations ${ }^{15,16}$. Of note, however, these aberrations are not completely eliminated. High-quality sample preparation is essential to ensure the best performance of any objective lens, and appropriate controls and corrections need to be applied for best practices ${ }^{3,5,9,17}$ (Box 2 and Supplementary Table 1).
The aberration corrections in an objective lens will have a profound effect on the interpretation of the microscopy data collected. Using a highly color-corrected lens will provide more precise measurements in experiments quantifying relative distance in multicolor three-dimensional (3D) imaging or colocalization (Fig. 2a). In the example provided, the extent of colocalization between channels depends on the objective correction, which, if not reported, could result in irreproducibility.

Numerical aperture (NA) is a critical characteristic of an objective lens because it indicates the amount of light that can be collected. Higher-NA lenses, under identical imaging conditions, produce brighter images than low-NA lenses ${ }^{9,11,18}$ (Fig. 2b). A low-NA lens will require longer exposure times or higher excitation light intensities to detect the fluorescence signal, which will impact temporal resolution, photobleaching and/or phototoxicity, leading to irreproducibility. Most importantly, the NA, and not the magnification, determines the resolving power of the system. Consequently, the NA of the objective lens will determine whether two objects of interest can be resolved or even detected under certain conditions (Fig. 2b,c). In the example, the high-NA objective lens produces sharper and more resolved images, improving the detection and detail of individual mitochondria.

Objective lenses are designed to work with a specific immersion medium (for example, air, oil, silicone oil, water or glycerol). There are many types of immersion oil, which vary in viscosity, refractive index and dispersion under different conditions. The type of immersion medium affects the performance of an objective lens, by inducing or minimizing spherical aberration and sometimes introducing fluorescence background signal. Therefore, two different immersion oils may result in different axial resolution and overall brightness of the image, thus affecting the ability to resolve objects axially or to detect the object of interest (Fig. 2d). The wrong immersion medium may also induce chromatic aberrations due to differences in dispersion ${ }^{19}$. Even though the type of immersion medium is critical to performance of the objective lens, it is overlooked in most methods sections. This reporting is especially critical for multi-immersion objective lenses, which can be used with multiple immersion media. 
a
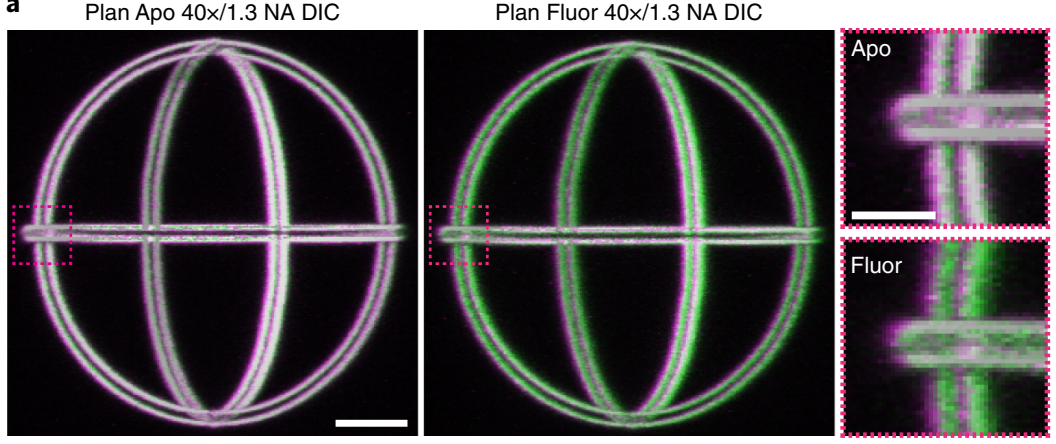

b

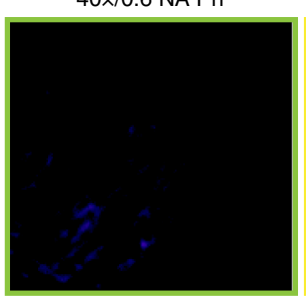

$40 \times / 0.95$ NA DIC
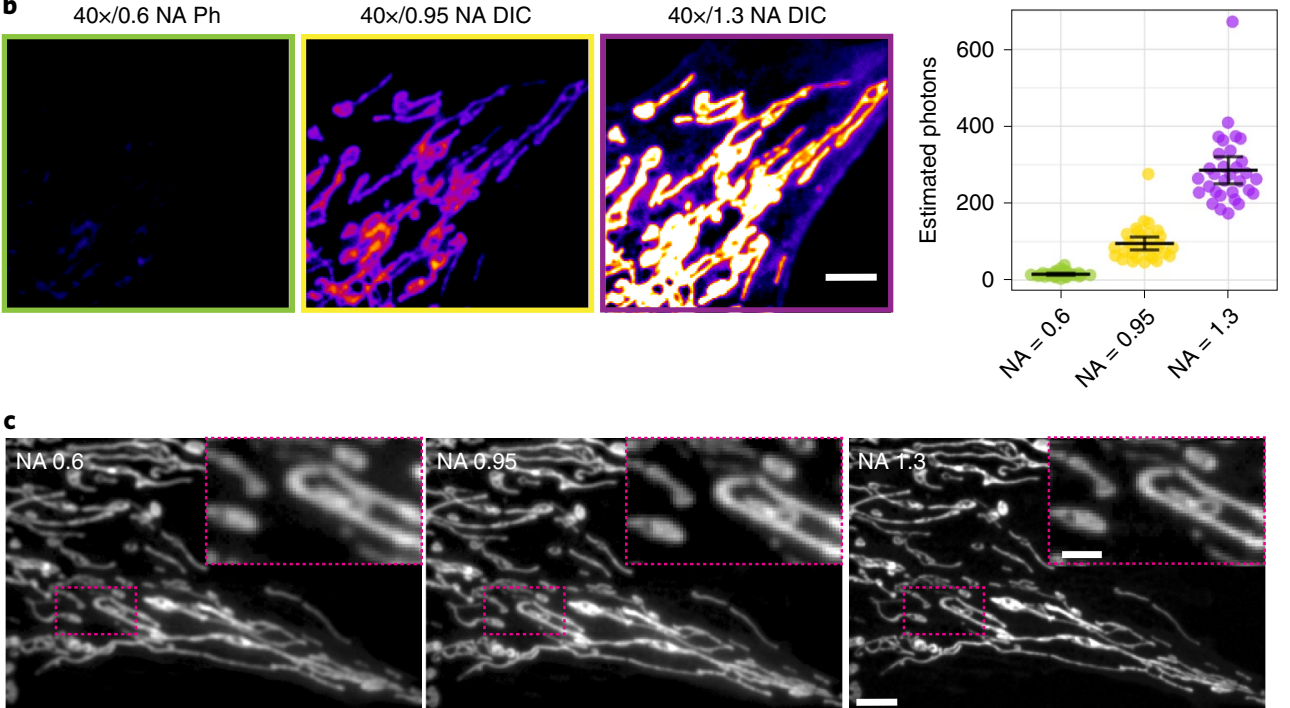

d
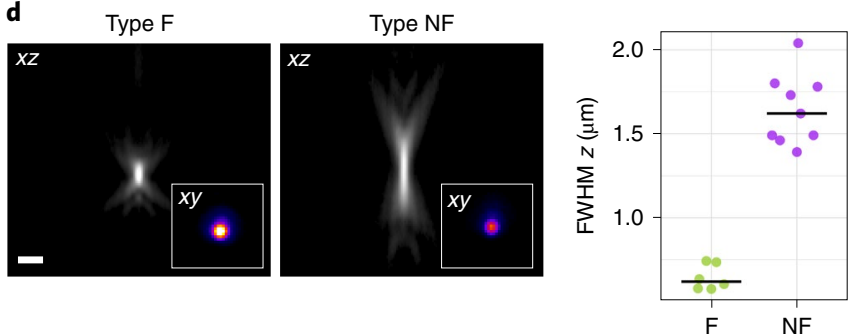

Fig. 2 | Objective lenses and immersion medium determine brightness and optical resolution and impact data interpretation. a, A 3D rendering of the 3D sphere pattern on the ArgoLight-SIM calibration slide acquired with two channels ( $488 \mathrm{~nm}$, green; $561 \mathrm{~nm}$, magenta) using either an Olympus Plan Apo 40x/1.3 NA DIC (left) or an Olympus Plan Fluor 40x/1.3 NA DIC (center) objective. Right, insets show the extent of overlap between signals (white). Overlay images are pseudocolored in green (488-nm excitation) and magenta (561-nm excitation). Scale bars, $5 \mu \mathrm{m}$. $\mathbf{b}$, Fluorescence images (using a Fire LUT) of BPAE cells stained with MitoTracker Red CMXRos acquired under identical imaging conditions using a Plan Fluor ELWD 40x/0.6 NA Ph2 (left), a Plan Apo 40x/0.95 NA DIC (center left) or a Plan Fluor 40x/1.3 NA oil-immersion DIC (center right) objective. Images are displayed at the same brightness/intensity scale. Right, violin plots of fluorescence intensity quantification of individual regions of interest. Long black bars indicate the mean fluorescence intensity for each population, and vertical bars indicate the s.d. Scale bar, $5 \mu \mathrm{m}$. $\mathbf{c}$, The same sample as in $\mathbf{b}$ imaged with the same objectives as in $\mathbf{b}$. Acquisition was optimized for each objective to obtain high-SNR images. Insets in each panel demonstrate the difference in resolution obtained when using objectives with different NA. Scale bars, $5 \mu \mathrm{m}$ (main panel) and $2 \mu \mathrm{m}$ (magnification). d, Representative $x z$ projections of $z$ stacks obtained by imaging 100-nm fluorescent beads passively adhered to \#1.5 HT coverslips and mounted with glycerol using a Nikon Plan Apo 100x/1.45 NA Ph3 objective with either Olympus Type F (left) or Nikon NF (center) immersion oil. Insets show the midplane displayed at the same scale using the Fire LUT. Right, dot plot illustrating the axial FWHM of individual beads quantified using the PSFj plugin when either the Type F or Type NF oil-immersion objective was used for imaging. The bars represent the population mean. Scale bar, $1 \mu \mathrm{m}$.

Detector. The detector used is critical in all aspects of quantitative microscopy. It impacts the likelihood that the fluorescence signal will be detected (sensitivity and noise level), the temporal resolution (frame rate) and the spatial resolution (digital resolution) of the system $^{20,21}$. As such, the choice of detector influences the capability to provide a conclusion faithful to the underlying biology. Thus, proper documentation ensures that similar observations can be made and similar conclusions can be drawn in different systems or experimental replicates.

The digital resolution of the system determines the ability to resolve the object of interest and will influence the downstream analysis workflow. In camera-based systems, it is determined by 
a

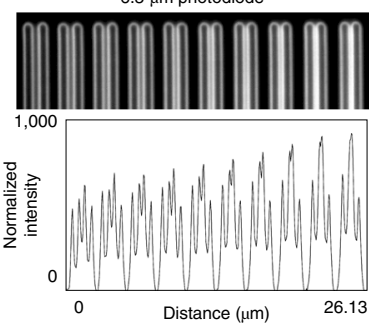

c

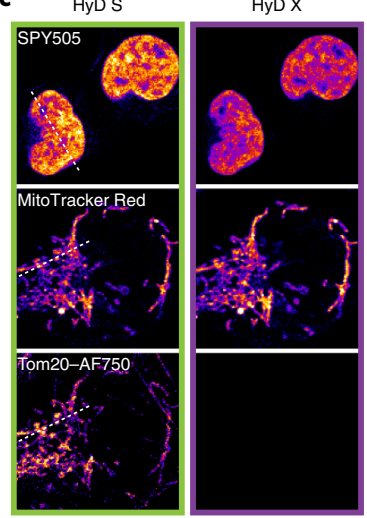

16- $\mu \mathrm{m}$ photodiode

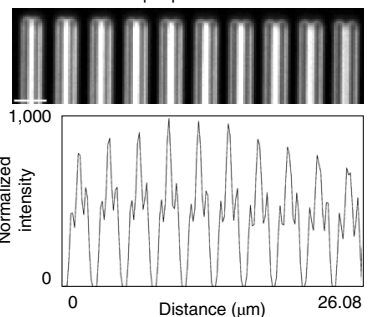

HyD R
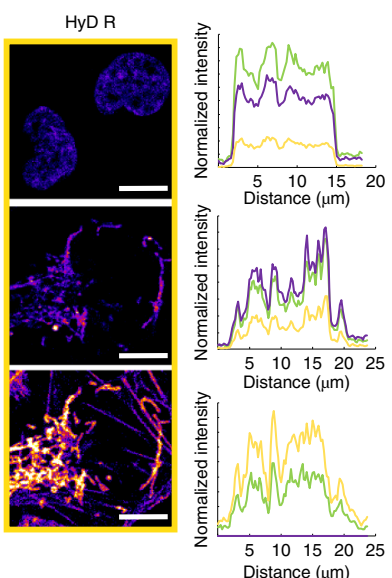

b

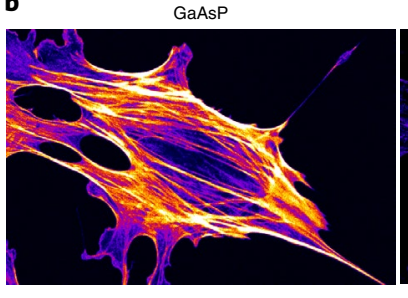

d
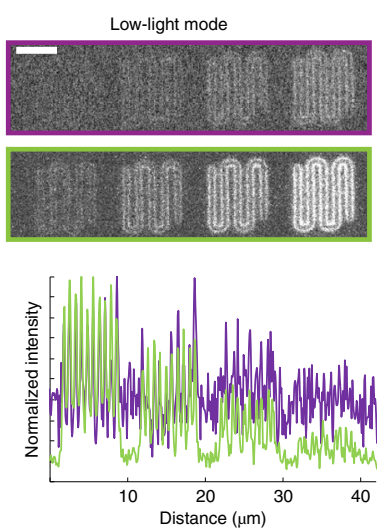

Multialkali
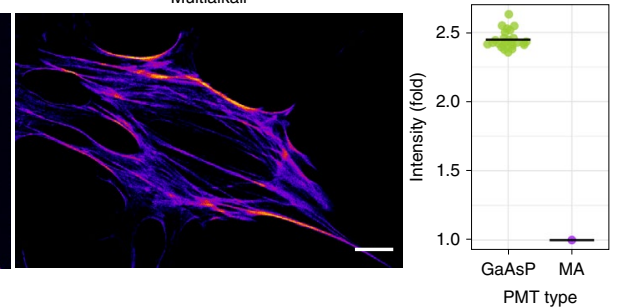

PMT type

Fig. 3 | The sensitivity of detectors varies with wavelength and determines the digital resolution and image quality. a, Fluorescence images of a pattern consisting of lines with incrementally increasing spacing on the ArgoLight-SIM calibration slide acquired with the same objective and a camera with either a $6.5-\mu \mathrm{m}$ (left) or 16- $\mu \mathrm{m}$ (right) photodiode size. Lower row, line intensity profile plots. Scale bar, $2.5 \mu \mathrm{m}$. $\mathbf{b}$, Fluorescence images (using a Fire LUT) of BPAE cells stained with Alexa Fluor 488 phalloidin acquired under identical imaging conditions with a single-point scanning confocal microscope using either a GaAsP (left) or multialkali PMT (MA; center) detector. Images are displayed with the same intensity scale. Right, fluorescence intensity quantification; bars represent the population mean. Scale bar, $10 \mu \mathrm{m}$. c, Fluorescence images (using a Fire LUT) of cultured cells prepared by Leica and imaged under identical conditions with a Leica Stellaris 8 Power HyD S (left column), Power HyD X (center-left column) or Power HyD R (center-right column) show a difference in intensity according to wavelength (right). Green, HyD S; purple, HyD X; yellow, HyD R. Scale bars, $10 \mu$ m. d, Fluorescence images of a pattern consisting of a repeating series of lines with progressively decreasing intensity (pattern C) on the ArgoLight-SIM calibration slide were acquired with an Andor Zyla sCMOS 4.2 plus camera under low-light conditions (left panels) or high-light conditions (right panels). Images were acquired under identical conditions in each case, with the exception of varying the amplifier gain amplification. Top left and top right, low gain; bottom left and bottom right, high gain. Bottom panels, plots of fluorescence intensities along a vertical line scan through the center of the pattern (purple, low gain amplification; green, high gain amplification). Scale bar, $5 \mu \mathrm{m}$.

the physical size of each photodiode (pixel) and the total magnification (Supplementary Exhibits 1-4). Data collected with two different cameras attached to the same microscope and acquired using the same objective lens can lead to fundamentally different interpretations of the results (Fig. 3a). The camera with a large photodiode size $(16 \mu \mathrm{m})$ cannot resolve the line pairs in the particular sample observed and instead creates a new pattern that does not correspond to the ground truth (aliasing). Pixel binning decreases the digital resolution and therefore must be reported ${ }^{20,21}$. Any additional magnification to the objective lens also needs to be reported to ensure measurement accuracy and precision, as this reduces light output (reduced SNR) and affects digital resolution (Supplementary Exhibits 1-4). In many cases, magnification changes are not accounted for in image calibrations and metadata, although not documenting them can lead to imprecision and irreproducibility in distance measurements (Box 2). This is true for any hardware component that is introduced manually into the lightpath, as it would not be recorded into the image metadata.

Image quality and SNR are greatly impacted by the sensitivity (quantum efficiency, QE) and noise level of the detector. These specifications determine the probability of detection and minimal detectable signal. The peak QE of detectors varies widely ${ }^{22}$ (70-95\% for cameras and 20-45\% for photomultiplier tubes, PMTs). Under otherwise identical conditions, a more sensitive detector will detect more light, producing a brighter image (Fig. 3b). Thus, the sensitivity and noise level of a particular detector will determine whether the signal can be detected at all and the dynamic range of the measurements, which in turn impacts their precision and accuracy. A less sensitive detector will require increased illumination intensity to detect the signal, which can induce photobleaching, fluorophore saturation and/or photodamage, leading to irreproducibility (Box 1).

Furthermore, the sensitivity of a detector varies with wavelength ${ }^{22}$. Many systems are equipped with various types of detectors that offer different sensitivity at different wavelengths, such as in single-point scanning confocal and multiphoton microscopes. In the example provided in Fig. $3 \mathrm{c}$, a researcher using a $\mathrm{HyD} \mathrm{X}$ detector (or another detector with similar specifications) to collect the emission of a near-infrared fluorophore would conclude that a protein of interest is not expressed in a particular cell type. Thus, the choice of detector in an experiment must be reported to ensure reproducibility.

The properties described above are inherent to a given detector; therefore, providing information on the specific manufacturer and model (for cameras) or the specific type of non-camera-based detector (for example, Multialkali PMT) will satisfy the minimal metadata requirements to ensure reproducibility. However, many detectors offer different acquisition settings that may affect 
the sensitivity, noise level and dynamic range of the detector, thus impacting the ability to detect the signal of interest. These settings should be documented, as they will impact reproducibility.

The readout mode or digitizer of the detector and the bit depth are good examples of such settings that critically impact image acquisition. Many cameras and other detectors offer a choice of which digitizer (and associated gain) to use during acquisition, which can be optimized depending on the amount of light generated by the sample. Higher gain settings result in higher intensity levels per electron than lower gain settings. Under low-light conditions (that is, for dim samples), a high gain setting may improve signal detection in comparison to a low gain setting (Fig. 3d). However, high gain settings also increase noise and reduce the dynamic range of the detector, so they are not ideal for bright samples as these samples will reach the saturation level sooner than when using low gain settings (Fig. 3d). Some cameras enable charge amplification (electron-multiplied charge-coupled device, EM-CCD ${ }^{20}$ ), thereby improving the detection of very dim signals for applications including single-molecule fluorescence. Thus, researchers should specify the particular camera settings or readout modes used during acquisition.

Microscope stand and relevant motorized components. Microscopy experiments usually require the acquisition of multidimensional data (for example, multiple planes, positions, channels, time points, etc.). The microscope stand and peripheral motorized components required depend on the goals of the experiment and the sample used (Supplementary Exhibits 1-5). The precision of the motorized components and how the software manages the image acquisition workflow may significantly impact the conclusions from the experiment, as they can introduce inaccuracies and limitations in measurements. Thus, knowledge of the hardware configuration and acquisition settings is required to collect precise, accurate and reproducible data. Consequently, the specifications of each component need to be verified as ideal for the application at hand, validated through appropriate controls (Box 2 and Supplementary Table 1) and accurately reported.

In multicolor experiments, the hardware used and whether the images are acquired sequentially or simultaneously will have a profound impact on several aspects of quantitative microscopy. Simultaneous acquisition will greatly increase the temporal resolution of the experiment but may also result in an increase in signal contamination between channels (Fig. 4a). In this case, simultaneous acquisition would lead to erroneous results.

Experiments that rely on the repeatability of positions, such as those tracking moving objects in multiple positions over time, are greatly impacted by the speed and precision of the lateral movement of motorized stages. In some cases, these parameters can be changed in the software (Supplementary Appendix), and overlooking and not documenting these settings will impact reproducibility. For example, the displacement between the centroids of stationary fluorescent beads imaged repeatedly in a multiple-position experiment can vary over fourfold depending on the precision settings of a motorized stage (Fig. 4b). This also applies to the axial location of the beads when acquiring a $z$ stack and will inevitably introduce error in the measurements (Fig. 4c). Furthermore, the order in which the acquisition is performed in a multidimensional experiment (for example, multicolor and $z$ stack) can also impact the results (Supplementary Appendix). Acquiring a $z$ stack of each wavelength instead of acquiring all the wavelengths at each $z$ step can lead to higher imprecision. Therefore, consideration of the focusing device used and the order in which the data are collected is critical during experimental design and must be reported (Fig. $4 \mathrm{c}$ and Box 2).

Furthermore, the $z$-step interval and total volume (and how it is selected) in a $z$ stack are critical to resolve objects in the axial dimension and need to be specified and reported. Larger step intervals than required (undersampling) can result in insufficient axial resolution. This will compromise downstream analysis and the conclusions of the experiment ${ }^{19}$ (Fig. 4d).

The success of a time-lapse imaging experiment depends on the ability to maintain focal and planar positioning and collect data at the appropriate temporal resolution and SNR to permit downstream image analysis without compromising the health of the sample. Irradiance is one of the key factors that affect sample health, as it can induce phototoxicity (Box 1). The effects of phototoxicity tend to be underestimated or overlooked, even though they are critical to properly interpret the observations $s^{4,23-26}$. Implementing and reporting hardware and acquisition settings that reduce irradiance is critical to allow meaningful comparisons between experiments (Supplementary Exhibits 1-4). Notably, the nominal time interval set in the software may not be consistent with the actual acquisition time interval, which depends on the shutter speed, delays due to the electronics that drive the acquisition and the acquisition software used. However, the timestamp found in the image metadata is likely accurate and should be used for quantification. Additionally, the actual average time interval and standard deviation should be included in the methods reported in a publication.

\section{Guidelines on reporting acquisition software}

Modern microscope systems are connected to a computer and controlled by acquisition software, which can be commercially available (for example, NIS Elements) or open source (for example, $\mu$ Manager). Software programming allows advanced automated image acquisition. Flexibility and integration depend on the specific software, the version (including relevant hotfixes) and available modules. Often, in the case of commercial packages, additional modules and/or upgrades have to be purchased individually, and the software can therefore vary substantially over time and between systems with almost identical hardware. It is critical to report software, modules and versions used. Any custom acquisition code should be

Fig. 4 | Data acquisition mode and instrument precision impact data reproducibility and interpretation. a, Fluorescence images of BPAE cells stained with DAPI and Alexa Fluor 488 phalloidin acquired either simultaneously in the same track (center right and right) or sequentially in two different tracks (left and center left). Scale bar, $15 \mu \mathrm{m}$. b. Dot plots of the displacement between centroids of 1- $\mu \mathrm{m}$ beads imaged repetitively with two different translational precision settings of a Nikon linear-encoded motorized stage (green, open, lower precision setting; purple, precise, highest precision setting). Multiple $x y$ positions were imaged repetitively over 15 loops. Bars represent the mean and s.e.m c, The xyz projections of TetraSpeck beads imaged by acquiring a $z$ stack in each channel (left) or both channels in each focal plane of the $z$ stack (center). Right, displacement plots of beads acquired using either the nosepiece Z drive (TiZ) or a PI piezo Z drive (piezo). Bars represent the mean and s.e.m. Scale bars, $2 \mu \mathrm{m}$. $\mathbf{d}$, Fluorescence images of a Convallaria section showing the midplane (left) and $x z$ and $y z$ projections from $z$ stacks acquired while varying the size of the $z$ step $(0.4 \mu \mathrm{m}, 2 \mu \mathrm{m}$ and $3 \mu \mathrm{m}$ ) (right). Scale bars, $10 \mu \mathrm{m}$. e, Same sample as in a imaged using a bidirectional scanning mode (left) or a unidirectional scanning mode (center). Inset images indicate a pronounced pixel shift in the image acquired using the bidirectional scanning mode when compared to the image acquired using the unidirectional scanning mode. Right, this shift can be corrected following calibration of the scanner. Scale bars, $5 \mu \mathrm{m}$ (main panel) and $1 \mu \mathrm{m}$ (magnification) .f, Same sample as in a acquired while varying the offset setting (top row, offset of $1.0 \mathrm{~V}$ (no offset); bottom row, offset of $-118 \mathrm{~V}$ ). Magnified views show processing steps to segment the mitochondria. Segmentation using images collected with improper offset settings results in identification of smaller objects (green arrowheads) and loss of objects (yellow arrowhead). Scale bars, $5 \mu \mathrm{m}$ (main panel and magnification). 
reported and made available to the scientific community, including the URL or website that contains information on its source and capabilities.

\section{Guidelines on reporting metadata specific to microscopy modality}

Optical sectioning techniques, such as confocal and multiphoton, are designed to enhance the contrast of a wide variety of biological specimens, including tissue sections or whole organs and organisms. They use vendor-specific methods to configure the light path, which may vary by instrument. Consequently, each microscopy modality will have dedicated hardware and software acquisition configurations that will impact results. In this section, we describe the metadata specific to single-point scanning confocal, spinning disk confocal and multiphoton microscopy. The metadata applicable to widefield microscopy are described in "Guidelines on reporting a

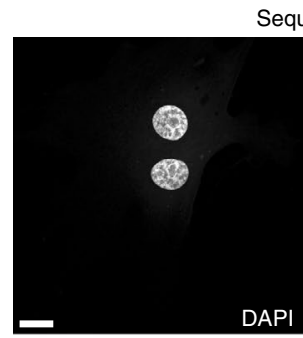

b

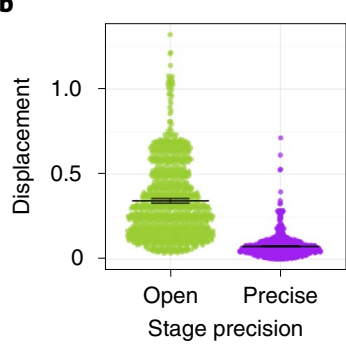

d
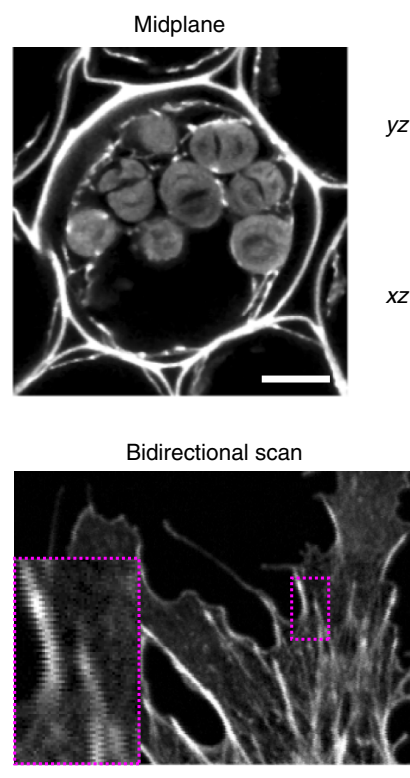

c

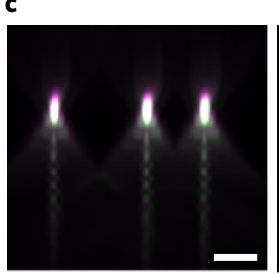

$z$ stack in each $\lambda$ $(z / \lambda)$
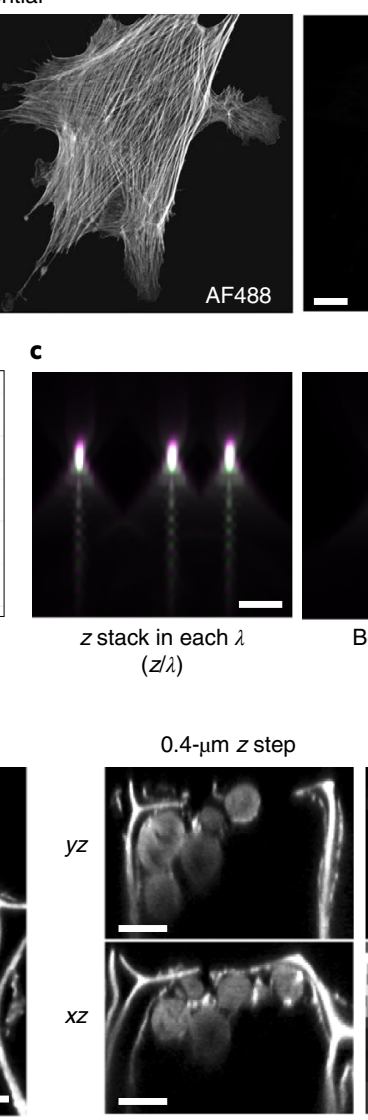

Unidirectional scan
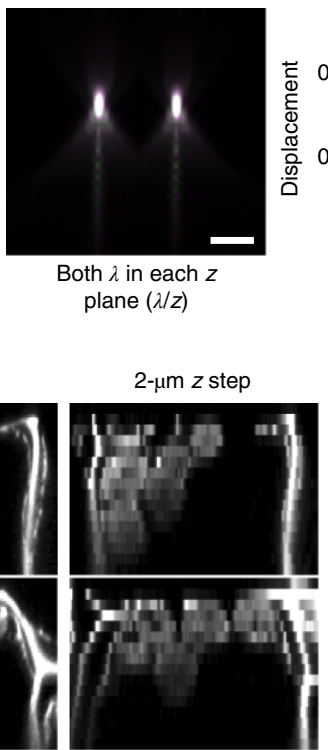

Simultaneous
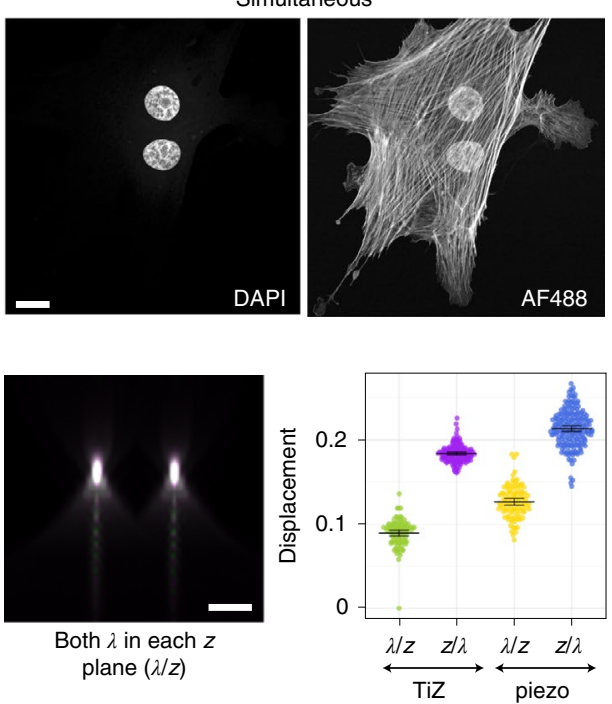

3- $\mu \mathrm{m} z$ step

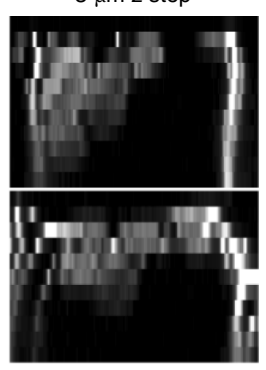

Bidirectional, calibrated

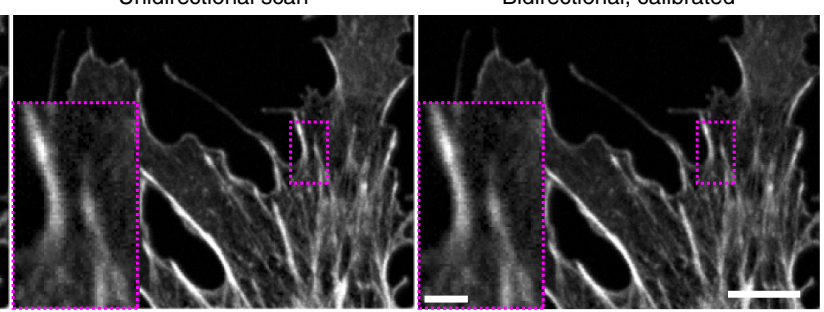

f
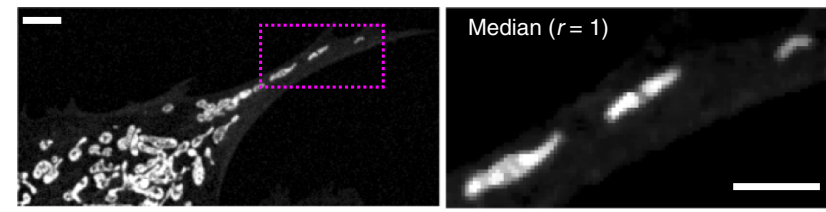

Mask
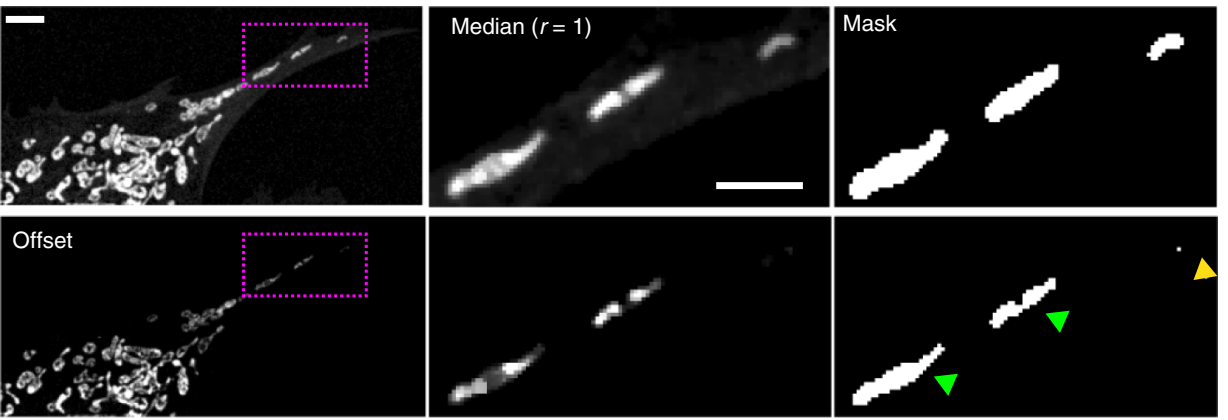
instrument data" and Supplementary Exhibit 1. Other microscopy modalities, such as light-sheet and super-resolution microscopy, require separate and specific guidelines and considerations and are beyond the scope of this Perspective.

Single-point scanning confocal microscopy. The light path in a laser scanning confocal microscope is more complex than that in a conventional widefield microscope and entails additional hardware and settings that will impact acquisition and, ultimately, the interpretation of the results.

The hardware components required for optical sectioning (scanning mechanism, illumination and wavelength selection configuration, pinhole and detection) are usually integrated into a scan unit. The specific hardware components vary between different vendors and even in models available from the same vendor. Reporting the scan unit model will specify the capabilities and limitations of each system, enabling other researchers to reproduce or interpret a particular experiment. Some scan units provide different options (for example, type of scanning mechanism and detectors) that can impact maximum acquisition frame rate, noise level and overall SNR and thus should be reported.

Although reporting the scan unit provides fixed information on the hardware configuration, there are many user-defined settings that contribute to image quality, quantification and conclusions and also need to be reported to ensure reproducibility (Supplementary Exhibit 2). Unfortunately, many of these settings are not well understood and are incorrectly applied and/or incompletely reported, which undoubtedly affects reproducibility. Below are some examples of the most commonly overlooked or misunderstood settings that have a great impact on the image and require reporting.

In a laser scanning confocal microscope, the digital resolution is not limited by a physical photodiode size. Instead, the detector ana$\log$ signal is digitized at regular intervals by the pixel clock. The final pixel size is determined by the total magnification, the pixel clock or frame size (how many intervals/pixels the scan area can be divided into) and the size of the scanned area (zoom factor). This offers flexibility but results in increased complexity, leading to irreproducibility if not properly reported. In many cases, researchers provide the frame size (for example, $1,024 \times 1,024$ ) instead of the actual pixel dimensions. This can be due to how the information is displayed in the acquisition software or to a poor understanding of the instrument. Regardless, the frame size alone does not provide crucial or reproducible information on the digital resolution of the image.

Most commercial laser scanning confocal microscopes can be used in uni- or bidirectional scanning modes. While the acquisition time can be reduced by half, bidirectional scanning may induce a pixel shift, especially at faster scan rates and higher zoom factors. This shift may not be apparent in the whole image, but careful inspection will show an offset between adjacent lines (Fig. 4e, left and inset, and Supplementary Appendix). Often, the scan mirrors can be carefully calibrated to reduce this pixel shift (Fig. 4e, right and inset), but reporting scanning directionality is still best practice.

A major setting that is often misused and under-reported is the offset. It may be tempting to adjust the offset to reduce fluorescence background and amplify the contrast for signal from the desired object. However, an improperly adjusted offset will lead to clipping of data intensities and removal of signal. If data clipping is substantial, entire structures within the sample can disappear from the image (Fig. 4f). Not reporting the offset will produce imprecise and conflicting quantitative measurements, such as the number of mitochondria per area, the width and area of each mitochondria and the overall intensity.

Another critical parameter to report is the pinhole diameter. It determines the thickness of the optical section, axial resolution and overall brightness and contrast. This information is typically given in Airy units (AU; Box 2 and Supplementary Table 2). It is important to note that the thickness of the optical section obtained by setting the pinhole to $1 \mathrm{AU}$ will vary with wavelength. The pinhole diameter (and wavelength, if stated in AU) should be reported.

The complexity in reporting the acquisition settings and configurations in a laser scanning confocal microscope is compounded by the lack of standardization of the nomenclature utilized by different vendors. For example, the adjustable voltage of the detector, which modulates the amount of gain or amplification of signal, can be referred to as master gain, HV (high voltage) and gain or even displayed as the percentage of amplification (Supplementary Appendix). In some acquisition software, adjustment of the scan rate (pixel dwell time) is provided on an arbitrary unit scale instead of in the appropriate units of microseconds per pixel or Hz. Caution is essential when reporting the scan rate/dwell time in these systems as inaccurate reporting would contribute to a lack of reproducibility (Supplementary Appendix). Current important initiatives to improve metadata standardization are listed in Supplementary Table 1.

Multiphoton microscopy. Multiphoton microscopes share most of the hardware components of a laser-scanning electron microscope and therefore will be impacted by many of the same parameters. A notable difference is the absence of a pinhole, as optical sectioning is provided by the localized excitation produced by the nearly simultaneous absorption of multiple photons at the focal plane ${ }^{27}$.

In this modality, a pulsed laser is necessary to achieve the high peak intensity required to excite fluorophores by nearly simultaneous absorption of multiple photons. The pulse length (femtosecond scale) and repetition frequency are important parameters as they determine the peak intensity ${ }^{28}$. Oftentimes tunable lasers are used; the laser model, excitation wavelength, pulse length and repetition frequency should be reported to ensure reproducibility.

Many multiphoton systems have two light paths to collect and detect the emitted light. The first light path coincides with the traditional confocal light path (scan unit), whereas the second light path uses specialized detectors (non-descanned detectors, NDDs) that collect emitted light more efficiently than detectors within the scan unit. The image produced by the NDD will have higher SNR than that from a scan unit detector, other things being equal, and therefore the specific hardware and configurations need to be specified to ensure reproducibility (Supplementary Exhibit 3).

Spinning disk confocal microscopy. In spinning disk confocal microscopes, the sample is scanned simultaneously through multiple pinholes of fixed diameter on a disk. The spacing of these pinholes greatly impacts optical sectioning capabilities. Closely spaced pinholes reduce optical sectioning and contrast, whereas disks with pinholes spaced farther apart improve optical sectioning in thicker specimens but reduce sensitivity and frame rate and require longer integration times or higher illumination levels to generate images with high SNR.

The manufacturer and model of the scan unit determine the design of the disk, the diameter of the pinholes and the spacing between them. Some scan units provide multiple pinhole size and spacing options. Reporting the specific pinhole size and spacing used in the experiment is critical to ensure reproducibility (Supplementary Exhibit 4).

\section{Guidelines on reporting sample preparation}

Sample preparation is critical in fluorescence microscopy and will greatly influence the quality of the final image and its quantification $^{29}$. There are many publications that focus on troubleshooting sample preparation and appropriate controls required for the interpretation of microscopy data in both fixed and live specimens ${ }^{3,5,9,17,29-33}$. Detailed sample preparation methods including fixation, permeabilization, labeling and mounting of the sample (Supplementary Exhibits 1-4) and validation steps should be 
documented when writing a manuscript. Here we highlight some examples of sample-related metadata that are critical for image acquisition in both fixed and live samples.

Of particular importance to report is the specific fluorophore used in any given experiment. Not only will the fluorophore's properties heavily dictate the experimental design and the hardware required to accomplish a particular experiment ${ }^{34-36}$, but, notably, the fluorophore can also impact the outcome of the experiment itself. This is especially true for specific variants of a fluorescent protein. Even if spectral properties are similar, characteristics such as brightness, photostability, monomeric quality and maturation time are variant specific ${ }^{37}$. The choice of fluorescent protein and how the fluorescent protein is attached to the target protein (C terminus, $\mathrm{N}$ terminus, or specific linker sequences and lengths) may alter the localization, concentration, lifetime and/or functionality of the protein of interest, leading to potentially erroneous measurements and different experimental outcomes ${ }^{38,39}$. Consequently, reporting GFP as the fluorophore (instead of sfGFP or mGFPmut3) does not provide sufficient information to reproduce a particular set of experiments and can result in critically different results. The original papers describing the specific fluorescent protein's development should also be cited if possible.

Sample optical properties contribute to the performance of the optics in the microscope and need to be documented. Objective lens corrections are engineered with a specific coverslip thickness in mind (usually $0.17 \mathrm{~mm}$ or grade no. 1.5). Use of a coverslip with a different thickness/grade can therefore negate those corrections ${ }^{13}$. Coverslip thickness tolerance (for example, $0.17 \pm 0.005 \mathrm{~mm}$ ) can be quite variable. Reporting the coverslip vendor and reference number therefore supports experimental reproducibility.

The mounting medium serves to homogenize the refractive index across the sample and match it to the requirements for the objective lens. The specifications of mounting media vary widely (refractive index, compatible fluorophores, curing, etc.). Mounting media can therefore impact the overall brightness, axial resolution and, in some cases, the final thickness of the sample, which will affect many aspects of quantification. Additionally, the refractive index of a mounting medium may change with time, and imaging in the same general window of time after mounting is recommended. Understanding how the mounting medium affects the sample and reporting its specifications will improve reproducibility.

Live-cell imaging enables the study of biological processes as they occur in real time though time-lapse imaging. In this application, maintaining the health of the sample should be the top priority to ensure rigor and reproducibility (Supplementary Exhibits 1-4 and Box 1). Most biological samples require an optimal temperature range to carry out biological processes. Precise control of the environmental conditions is critical to ensure normal cell physiology and function, including growth rate and molecular dynamics. The hardware used to maintain temperature, gas and humidity vary widely and will offer different stability and levels of control over these conditions. Thus, a description of the specific environmental conditions and how these are maintained will greatly improve accuracy, precision and reproducibility. The specific imaging medium and any additional components that may decrease phototoxicity or photobleaching (for example, antioxidants and reactive oxygen species scavengers) should be reported. Some components of the medium such as phenol red, fetal bovine serum, riboflavins and vitamins can produce substantial fluorescence background signal, limiting the ability to detect the signal of interest and impacting the accuracy and precision of quantitative measurements. Additionally, the concentration of a fluorescent dye and the solvent used or transfection reagents and expression of fluorescent protein fusions ${ }^{37}$ in a live sample may affect cell function ${ }^{40-42}$, induce synergistic effects with the conditions used (for example, drug treatments), and therefore impact reproducibility.
There is a wealth of publications offering insight on live-cell imaging that researchers can consult to better understand, optimize and troubleshoot aspects related specifically to this application $^{4,18,33,43,44}$.

\section{Notes on method validation}

Understanding the capabilities and limitations of the particular application is essential in designing a successful microscopy experiment, as it enables researchers to identify the sources of measurement imprecision and mitigate its effects. In addition to carefully reporting microscopy methods, authors should aim to report critical steps for validation of the methodology. Box 2 describes some of the important validation steps that should be included in any experimental design and in the methods section, and Supplementary Table 1 lists selected resources for method validation. There are several outstanding publications that provide more information on method validation approaches and protocols ${ }^{3,5,17}$. Additionally, several initiatives in the microscopy field focus on the importance of quality control and instrument performance assessment to validate microscopy methods (Supplementary Table 1).

\section{Notes on image format and processing and their impact on metadata}

Image processing is used to enhance visualization and extract information from the digital image. While image processing is important for gleaning useful information (for example, during segmentation), it can irreversibly change the intensity levels and introduce nonlinear changes in shape and intensity across the image, compromising accurate quantification. Thus, all imaging processing steps and software settings must be reported ${ }^{45,46}$.

Most acquisition software options include image processing shortcuts to enhance image contrast (for example, haze reduction or smoothing) or other aspects of the image automatically or with a few convenient clicks of a button, often intended for presentation purposes while compromising the quantitative integrity of the image. Importantly, the resulting processed image is generally no longer faithful to the raw data collected, compromising reproducibility and accuracy in subsequent measurements, especially if the raw data are not saved alongside the processed image. Researchers should pay careful attention to software controls/modules used and ask experts (vendors or core scientists) for guidance in image processing. Most critically, the raw data image should always be saved.

Another aspect to consider is the file format used to acquire and export/save the images. Most commercial software produces a raw, uncompressed proprietary image format that contains all accompanying metadata recorded by the system. However, images can be exported in other file formats that may compress or otherwise scale or filter the data, reduce bit depth, alter the intensity levels acquired by the detector and lose the metadata, often irreversibly (for example, jpeg, mov, AVI). Ensuring a copy of the raw image in its original suggested proprietary file format is saved and backed up before converting to or exporting as a different file format is absolutely critical for quantitative microscopy. No matter how tempting it is to work with small file sizes, a compromise, often significant, has been made, even if unintentionally.

\section{Resources for reporting microscopy methods}

The examples provided in this Perspective serve as cautionary tales of how the lack of proper methods reporting can lead to conflicting data interpretation and thus irreproducibility. To facilitate proper microscopy methods reporting, we have assembled comprehensive checklists for the most common fluorescence light microscopy modalities (Supplementary Exhibits 1-4). Each checklist contains essential and recommended metadata to include in the methods section for any manuscript presenting data that were acquired using these modalities. Common examples are provided for each term, 
and a reference text example of a methods section write-up is also included after the checklist.

Additionally, to help researchers simplify and customize the essential metadata list according to their specific imaging and experimental design, we developed a microscopy metadata checklist generator tool (MicCheck). This tool guides researchers through simple questions related to their imaging choices and dynamically generates a checklist of essential and optional metadata that can then be copied and pasted into a text editor or downloaded in pdf format (Supplementary Exhibit 5). MicCheck is hosted online at https://rebecca-senft.shinyapps.io/MicCheck/, and relevant source files are available at the GitHub repository at https://github.com/ rsenft1/MicCheck. In addition to online use, by downloading and modifying the example text file, core facilities or laboratories are also able to create their own versions of MicCheck with custom metadata examples specific to their microscope systems.

These checklists can also be used by researchers when designing imaging experiments to ensure that the relevant hardware and settings are properly identified and configured. They can also be used for reporting imaging methods, including during manuscript preparation or in the experimental design sections of funding proposals. Additionally, the checklists can be used by editors and reviewers to ensure sufficient detail is included to consider the experiments rigorous and reproducible.

Finally, for education and to help improve the adoption of these guidelines, we provide a visual infographic that can be printed and displayed in laboratories, core facilities or microscopy rooms to aid, remind and encourage researchers to compose detailed methods sections in their publications (Extended Data Fig. 1). The poster summarizes the essential metadata described in the checklists and the figures, depicting examples of how these hardware and settings can impact image quality and reproducibility.

\section{Recommendations to improve methods reporting}

While the description of minimal microscopy reporting guidelines is an essential first step toward improving methods reporting, it is not sufficient to solve the microscopy reproducibility crisis. The imaging scientific community needs to work together in a coordinated effort to improve methods reporting and the overall quality and reproducibility of image-based research, including researchers, imaging scientists, institutions, granting agencies, scientific journals and vendors. More rigorous and reproducible science results in better use of resources, improved data integrity and, therefore, fewer retracted publications and the ability to extend research by mining published results. Thus, there is a need to establish standards and provide resources to educate, inform and improve microscopy methods metadata reporting. Greater awareness and education will improve how microscopy data are collected, shared, validated, analyzed and reported.

Role for researchers. Researchers can contribute to improvement in microscopy methods reporting by (1) improving their knowledge of the microscopy techniques and appropriate validation steps used throughout their research; (2) upholding and requiring guidelines and recommendations set by the imaging community when reviewing manuscripts and grant applications; (3) involving imaging scientists during all steps of the research process, from experimental setup to data interpretation and manuscript preparation; (4) acknowledging core facilities and imaging scientists or considering collaborations that lead to authorship, as a way to enable the critical role core facilities fulfill in support of the scientific community; and (5) using data repositories to improve transparency and reproducibility and enhance data sharing ${ }^{47}$ (Supplementary Table 1).

Role for imaging scientists and core staff. Imaging scientists, especially those in core facilities, have a key role by sharing their technical expertise and providing intellectual contributions in all aspects of image-based science. In addition, they serve an educational mission by disseminating resources and tools to improve image-based research. Educating researchers on the importance of appropriate methods reporting will encourage best practices and participation by everyone. While core staff and imaging scientists have a critical educational role, this is not a substitute for formal training of graduate students and other researchers in proper metadata collection and reporting. Such training can take place at home institutions and in summer or other intensive programs focused on microscopy methodology and will greatly improve education and awareness broadly.

Imaging scientists are encouraged to stay informed about resources on education, rigor and reproducibility and to contribute to the development of guidelines and standards by many initiatives in the imaging community ${ }^{6,48-50}$. These initiatives (including those in Supplementary Table 1, as well as many others) can help in this task by presenting the resources in conferences, workshops and other educational initiatives they support.

Role for scientific journals and funding agencies. Scientific journals are urged to update their instructions to authors and have them reviewed by the imaging community, to ensure that adequate microscopy metadata information is included in the materials and methods. Removing word count limits from the methods section will allow authors to fully describe microscopy image collection and analysis, which should be written and reviewed with the same rigor as the entire paper. If space constraints cannot be adjusted, detailed methods descriptions can be included in supplementary information.

Encouraging or requiring proper recognition of the contribution of imaging scientists, as appropriate (acknowledgment or authorship), will facilitate researchers working closely with imaging scientists as standard practice. Incorporating imaging scientists in the peer review process, perhaps as technical experts, would help ensure that the experimental design, methodology and reporting of microscopy data support the conclusions of the manuscript.

Lastly, requiring appropriate analysis and quantification of imaging data (in addition to 'representative images') is long overdue. Quantification and validation of microscopy data will reduce bias and irreproducibility as well as the publication of artifactual results.

\section{Conclusion}

Rigorous and unbiased experimental design and analysis workflows are critical to provide accurate insight into the biological process under investigation ${ }^{3,5,9,17}$. Sample preparation, choice of instrument and related hardware, and image acquisition parameters (that is, metadata) have a profound effect on the image data validity and interpretation and therefore must be reported in the methods section of a published manuscript. However, microscopy methods reporting is often overlooked throughout the peer review process. In this work, we describe specific examples that highlight how lack of reporting of comprehensive information can affect the integrity and reproducibility of microscopy results. We present guidelines, checklists and resources to help researchers identify the critical metadata that should be included in their methods section, according to their specific experiment. The goal is not to put an unnecessary burden on researchers, but rather to help give readers of research papers enough information to assess the validity of biological findings and reproduce those findings independently. We hope that these reporting guidelines become second nature when carrying out microscopy experiments and reporting microscopy data and that implementing these guidelines will help improve the design of future experiments. We also hope that these guidelines provide a starting point for journal editors and peer reviewers when assessing microscopy data. 
We recognize that addressing microscopy reproducibility is a complex, multifaceted issue that will require an ongoing and coordinated effort from everyone involved in scientific research. In particular, we recognize the contributions from core facilities and imaging scientists who are uniquely positioned to assist researchers in addressing these challenges by educating and supporting the scientific community ${ }^{3,5,6,9,17,44,45,48-52}$ (Supplementary Table 1). While this Perspective has focused on reporting of microscopy methodology, we emphasize that appropriate method validation and experimental design are critical to ensure the quality of quantitative microscopy data and the continued progress of feasible, rigorous and reproducible image-based science.

Reporting Summary. Further information on research design is available in the Nature Research Reporting Summary linked to this article.

\section{Data availability}

The raw image data used in the figures are publicly available in OMERO at https://omero.hms.harvard.edu/webclient/userdata/?e xperimenter $=1553$.

\section{Code availability}

Relevant source files for MicCheck are available at the GitHub repository at https://github.com/rsenft1/MicCheck.

Received: 11 February 2021; Accepted: 15 April 2021;

Published online: 7 June 2021

\section{References}

1. Marqués, G., Pengo, T. \& Sanders, M. A. Imaging methods are vastly underreported in biomedical research. eLife https://doi.org/10.7554/ eLife.55133 (2020).

2. Jonkman, J., Brown, C. M., Wright, G. D., Anderson, K. I. \& North, A. J. Guidance for quantitative confocal microscopy. Nat. Protoc. https://doi. org/10.1038/s41596-020-0307-7 (2020).

3. Jost, A. P. \& Waters, J. C. Designing a rigorous microscopy experiment: validating methods and avoiding bias. J. Cell Biol. 218, 1452-1466 (2019).

4. Kiepas, A., Voorand, E., Mubaid, F., Siegel, P. M. \& Brown, C. M. Optimizing live-cell fluorescence imaging conditions to minimize phototoxicity. J. Cell Sci. https://doi.org/10.1242/jcs.242834 (2020).

5. Wait, E. C., Reiche, M. A. \& Chew, T. L. Hypothesis-driven quantitative fluorescence microscopy - the importance of reverse-thinking in experimental design. J. Cell Sci. https://doi.org/10.1242/jcs.250027 (2020).

6. Hammer, M. et al. Towards community-driven metadata standards for light microscopy: tiered specifications extending the OME model. Preprint at bioRxiv https://doi.org/10.1101/2021.04.25.441198 (2021).

7. Reigoto, A. M., Andrade, S. A., Seixas, M. C. R. R., Costa, M. L. \& Mermelstein, C. A comparative study on the use of microscopy in pharmacology and cell biology research. PLoS ONE 16, e0245795 (2021).

8. Mubaid, F. et al. Fluorescence microscope light source stability. Histochem Cell Biol. 151, 357-366 (2019).

9. North, A. J. Seeing is believing? A beginners' guide to practical pitfalls in image acquisition. J. Cell Biol. 172, 9-18 (2006).

10. Stack, R. F. et al. Quality assurance testing for modern optical imaging systems. Microsc. Microanal. 17, 598-606 (2011).

11. Waters, J. C. Accuracy and precision in quantitative fluorescence microscopy. J. Cell Biol. 185, 1135-1148 (2009).

12. Goodwin, P. C. in Methods in Cell Biology Vol. 114 (eds Sluder, G. \& Wolf, D. E.) 369-385 (Academic Press, 2013).

13. Keller, H. E. in Handbook of Biological Confocal Microscopy (ed. Pawley, J.) 207-220 (Springer, 2006)

14. Ross, S. T., Allen, J. R. \& Davidson, M. W. Practical considerations of objective lenses for application in cell biology. Methods Cell. Biol. 123, 19-34 (2014)

15. Cole, R. W. et al. International test results for objective lens quality, resolution, spectral accuracy and spectral separation for confocal laser scanning microscopes. Microsc. Microanal. 19, 1653-1668 (2013).

16. Cole, R. W., Jinadasa, T. \& Brown, C. M. Measuring and interpreting point spread functions to determine confocal microscope resolution and ensure quality control. Nat. Protoc. 6, 1929-1941 (2011).

17. Lee, J. Y. \& Kitaoka, M. A beginner's guide to rigor and reproducibility in fluorescence imaging experiments. Mol. Biol. Cell 29, 1519-1525 (2018).
18. Waters, J. C. Live-cell fluorescence imaging. Methods Cell. Biol. 81, 115-140 (2007)

19. Diel, E. E., Lichtman, J. W. \& Richardson, D. S. Tutorial: avoiding and correcting sample-induced spherical aberration artifacts in $3 \mathrm{D}$ fluorescence microscopy. Nat. Protoc. 15, 2773-2784 (2020).

20. Lambert, T. J. \& Waters, J. C. Assessing camera performance for quantitative microscopy. Methods Cell. Biol. 123, 35-53 (2014).

21. Murphy, D. \& Davidson, M. W. Fundamentals of Light Microscopy \& Electronic Imaging 2nd edn (Wiley-Blackwell, 2012).

22. Art, J. in Handbook of Biological Confocal Microscopy (ed. Pawley, J.) 251-264 (Springer, 2006).

23. Dixit, R. \& Cyr, R. Cell damage and reactive oxygen species production induced by fluorescence microscopy: effect on mitosis and guidelines for non-invasive fluorescence microscopy. Plant J. 36, 280-290 (2003).

24. Ettinger, A. \& Wittmann, T. Fluorescence live cell imaging. Methods Cell. Biol. 123, 77-94 (2014)

25. Icha, J., Weber, M., Waters, J. C. \& Norden, C. Phototoxicity in live fluorescence microscopy, and how to avoid it. BioEssays https://doi. org/10.1002/bies.201700003 (2017).

26. Magidson, V. \& Khodjakov, A. Circumventing photodamage in live-cell microscopy. Methods Cell. Biol. 114, 545-560 (2013).

27. Benninger, R. K. \& Piston, D. W. Two-photon excitation microscopy for the study of living cells and tissues. Curr. Protoc. Cell Biol. Ch. 4, Unit 4.11.11-4.11.24 (2013)

28. Denk, W., Piston, D. \& Webb, W. in Handbook of Biological Confocal Microscopy (ed. Pawley, J.) 535-549 (Springer, 2006).

29. Burry, R. Immunocytochemistry: A Practical Guide for Biomedical Research (Springer, 2010).

30. Allan, V. Protein Localization by Fluorescence Microscopy: A Practical Approach (Oxford University Press, 2000).

31. Allen, J. R., Ross, S. T. \& Davidson, M. W. Sample preparation for single molecule localization microscopy. Phys. Chem. Chem. Phys. 15, 18771-18783 (2013).

32. Bacallao, R., Kiai, K. \& Jesaitis, L. in Handbook of Biological Confocal Microscopy 3rd edn (ed. Pawley, J.) 311-323 (Springer, 2013).

33. Specht, E. A., Braselmann, E. \& Palmer, A. E. A critical and comparative review of fluorescent tools for live-cell imaging. Annu. Rev. Physiol. 79, 93-117 (2017)

34. Heppert, J. K. et al. Comparative assessment of fluorescent proteins for in vivo imaging in an animal model system. Mol. Biol. Cell 27, 3385-3394 (2016).

35. Lambert, T. J. FPbase: a community-editable fluorescent protein database. Nat. Methods 16, 277-278 (2019).

36. Shaner, N. C. Fluorescent proteins for quantitative microscopy: important properties and practical evaluation. Methods Cell. Biol. 123, 95-111 (2014).

37. Cranfill, P. J. et al. Quantitative assessment of fluorescent proteins. Nat. Methods 13, 557-562 (2016).

38. Landgraf, D., Okumus, B., Chien, P., Baker, T. A. \& Paulsson, J. Segregation of molecules at cell division reveals native protein localization. Nat. Methods $\mathbf{9}$, 480-482 (2012).

39. Montecinos-Franjola, F., Bauer, B. L., Mears, J. A. \& Ramachandran, R. GFP fluorescence tagging alters dynamin-related protein 1 oligomerization dynamics and creates disassembly-refractory puncta to mediate mitochondrial fission. Sci. Rep. 10, 14777 (2020).

40. Alford, R. et al. Toxicity of organic fluorophores used in molecular imaging: literature review. Mol. Imaging 8, 7290.2009 .00031 (2009).

41. Spracklen, A. J., Fagan, T. N., Lovander, K. E. \& Tootle, T. L. The pros and cons of common actin labeling tools for visualizing actin dynamics during Drosophila oogenesis. Dev. Biol. 393, 209-226 (2014).

42. Yang, Z. et al. Cyclooctatetraene-conjugated cyanine mitochondrial probes minimize phototoxicity in fluorescence and nanoscopic imaging. Chem. Sci. 11, 8506-8516 (2020).

43. Brown, C. M. Fluorescence microscopy-avoiding the pitfalls. J. Cell Sci. 120 , 1703-1705 (2007)

44. Frigault, M. M., Lacoste, J., Swift, J. L. \& Brown, C. M. Live-cell microscopytips and tools. J. Cell Sci. 122, 753-767 (2009).

45. Aaron, J. S. \& Chew, T. L. A guide to accurate reporting in digital image processing: can anyone reproduce your quantitative analysis? J. Cell Sci. https://doi.org/10.1242/jcs.254151 (2021)

46. Miura, K. \& Nørrelykke, S. F. Reproducible image handling and analysis. EMBO J. 40, e105889 (2021).

47. Ellenberg, J. et al. A call for public archives for biological image data. Nat. Methods 15, 849-854 (2018).

48. Nelson, G. et al. QUAREP-LiMi: a community-driven initiative to establish guidelines for quality assessment and reproducibility for instruments and images in light microscopy. Preprint at https://arxiv.org/abs/2101.09153 (2021).

49. Kunis, S., Hänsch, S., Schmidt, C., Wong, F. \& Weidtkamp-Peters, S. OMERO mde in a use case for microscopy metadata harmonization: facilitating FAIR principles in practical application with metadata annotation tools. Preprint at https://arxiv.org/abs/2103.02942 (2021). 
50. Mische, S. M. et al. A review of the scientific rigor, reproducibility, and transparency studies conducted by the ABRF research groups. J. Biomol. Tech. 31, 11-26 (2020).

51. Heddleston, J. M., Aaron, J. S., Khuon, S. \& Chew, T. L. A guide to accurate reporting in digital image acquisition-can anyone replicate your microscopy data? J. Cell Sci. https://doi.org/10.1242/jcs.254144 (2021).

52. Waters, J. C. A novel paradigm for expert core facility staff training. Trends Cell Biol. 30, 669-672 (2020).

53. Patterson, G. H. \& Piston, D. W. Photobleaching in two-photon excitation microscopy. Biophys. J. 78, 2159-2162 (2000)

54. Wang, E., Babbey, C. M. \& Dunn, K. W. Performance comparison between the high-speed Yokogawa spinning disc confocal system and single-point scanning confocal systems. J. Microsc. 218, 148-159 (2005).

55. Onukwufor, J. O. et al. Quantification of reactive oxygen species production by the red fluorescent proteins KillerRed, SuperNova and mCherry. Free Radic. Biol. Med. 147, 1-7 (2020).

56. Schmidt, G. W., Cuny, A. P. \& Rudolf, F. Preventing photomorbidity in long-term multi-color fluorescence imaging of Saccharomyces cerevisiae and $S$. pombe. G3 10, 4373-4385 (2020).

57. Hoebe, R. A., Van der Voort, H. T., Stap, J., Van Noorden, C. J. \& Manders, E. M. Quantitative determination of the reduction of phototoxicity and photobleaching by controlled light exposure microscopy. J. Microsc. 231, 9-20 (2008).

58. Nishigaki, T., Wood, C. D., Shiba, K., Baba, S. A. \& Darszon, A. Stroboscopic illumination using light-emitting diodes reduces phototoxicity in fluorescence cell imaging. Biotechniques 41, 191-197 (2006).

59. Theer, P., Mongis, C. \& Knop, M. PSFj: know your fluorescence microscope. Nat. Methods 11, 981-982 (2014).

60. Hodgson, L., Shen, F. \& Hahn, K. Biosensors for characterizing the dynamics of Rho family GTPases in living cells. Curr. Protoc. Cell Biol. Chapter 14, Unit 14.11.11-26 (2010).

61. Kraus, B., Ziegler, M. \& Wolff, H. in Modern Research and Educational Topics in Microscopy Vol. 2.1 (eds Méndez-Vilas, A. \& Díaz, J.) 863-872 (Formatex, 2007).

62. Model, M. A. Intensity calibration and shading correction for fluorescence microscopes. Curr. Protoc. Cytom. Chapter 10, Unit 10.14 (2006).

63. Model, M. A. \& Blank, J. L. Concentrated dyes as a source of two-dimensional fluorescent field for characterization of a confocal microscope. J. Microsc. 229, 12-16 (2008).

64. Wolf, D. E., Samarasekera, C. \& Swedlow, J. R. Quantitative analysis of digital microscope images. Methods Cell. Biol. 114, 337-367 (2013).

65. Diaspro, A., Chirico, G., Usai, C., Ramoino, P. \& Dobrucki, J. in Handbook of Biological Confocal Microscopy (ed. Pawley, J.) 690-702 (Springer, 2006).

66. Vicente, N. B., Zamboni, J. E. D., Adur, J. F., Paravani, E. V. \& Casco, V. H. Photobleaching correction in fluorescence microscopy images. J. Phys. Conf. Ser. 90, 012068 (2007)

\section{Acknowledgements}

We thank C. Strambio De Castillia and C.M. Brown for helpful discussions and comments during manuscript preparation, and we thank R. Cole, A. Laude, G. Nelson,
R. Nitschke and W. Salmon for feedback on the manuscript. We thank A. Vettiger (Bernhardt laboratory, Harvard Medical School) and G. Miner (Cohen laboratory, University of North Carolina at Chapel Hill) for the bacterial and U2OS samples, respectively. We thank Leica Microsystems for their help collecting the images in Fig. 3c and for supplying the custom six-color sample. We thank our microscopy core facility staff and users of the Microscopy Resources on the North Quad (MicRoN) Core at Harvard Medical School, the Duke University Light Microscopy Core Facility, the Neuroscience Microscopy Core Facility (RRID: SCR_019060) of the University of North Carolina School of Medicine and the University Imaging Centers of the University of Minnesota (RRID: SCR_020997). Imaging was performed at the MicRoN Core at Harvard Medical School and at the UNC Neuroscience Microscopy Core Facility supported, in part, by funding from the NIH-NINDS Neuroscience Center Support Grant P30 NS045892 and the NIH-NICHD Intellectual and Developmental Disabilities Research Center Support Grant P50 HD103573. M.S.I. is supported by an Imaging Scientist grant number 2019-198107 from the Chan Zuckerberg Initiative DAF, an advised fund of the Silicon Valley Community Foundation. M.A.S. is supported by an Imaging Scientist grant from the Chan Zuckerberg Initiative. R.A.S. was supported by National Institutes of Health Grant F31-NS-108406.

\section{Author contributions}

P.M.L., L.A.C. and M.S.I. wrote the manuscript, including the boxes, supplementary information and checklist content. R.A.S. created MicCheck and contributed to manuscript editing and Supplementary Table 2. R.S. collected data for Figs. $2 b-d$ and $4 b$, analyzed data for Fig. $2 \mathrm{~b}$ and created the Supplementary Appendix. T.J.R.-E. collected data for Figs. 1d, 2a and 4a,c. D.P.K. collected and prepared Fig. 1b, the legend for Fig. $1 \mathrm{~b}$ and methods. P.M.L. collected data for Figs. 1a, 3 and $4 \mathrm{~d}-\mathrm{f}$, analyzed data for Figs. 1b, 3 and $4 \mathrm{~b}, \mathrm{c}$, and prepared the figures and Extended Data Fig. 1. M.S.I., D.P.K. and P.K. prepared and formatted the checklists. G.M. and M.A.S. contributed to manuscript writing and editing. T.P., B.R.C. and Y.-S.G. contributed to manuscript organization and editorial formatting.

\section{Competing interests}

The authors declare no competing interests.

\section{Additional information}

Extended data is available for this paper at https://doi.org/10.1038/s41592-021-01156-w. Supplementary information The online version contains supplementary material available at https://doi.org/10.1038/s41592-021-01156-w.

Correspondence should be addressed to P.M.L.

Peer review information Nature Methods thanks Heather Brown-Harding, Jay Unruh and the other, anonymous, reviewer(s) for their contribution to the peer review of this work. Rita Strack was the primary editor on this article and managed its editorial process and peer review in collaboration with the rest of the editorial team.

Reprints and permissions information is available at www.nature.com/reprints. Publisher's note Springer Nature remains neutral with regard to jurisdictional claims in published maps and institutional affiliations.

(c) Springer Nature America, Inc. 2021 


\section{naturemethods Best practices and tools for reporting reproducible microscopy methods}

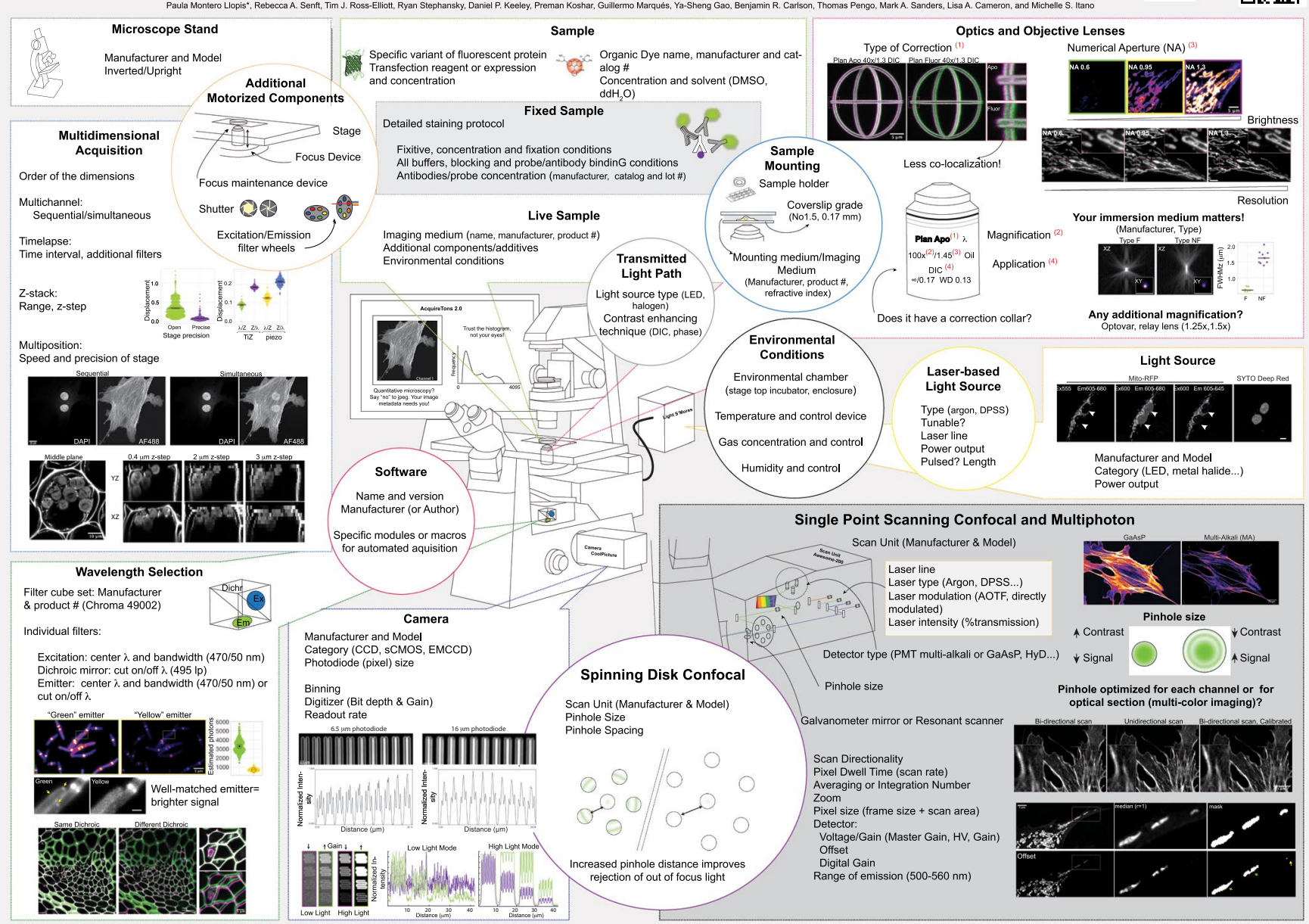

Extended Data Fig. 1 | 'Best practices and tools for reporting reproducible fluorescence microscopy methods' infographic. Downloadable visual representation of the guidelines on microscopy method reporting proposed in this Perspective. 


\section{Reporting Summary}

Nature Research wishes to improve the reproducibility of the work that we publish. This form provides structure for consistency and transparency in reporting. For further information on Nature Research policies, see our Editorial Policies and the Editorial Policy Checklist.

\section{Statistics}

For all statistical analyses, confirm that the following items are present in the figure legend, table legend, main text, or Methods section.

n/a Confirmed

Х $\square$ The exact sample size $(n)$ for each experimental group/condition, given as a discrete number and unit of measurement

Х $\square$ A statement on whether measurements were taken from distinct samples or whether the same sample was measured repeatedly

Х The statistical test(s) used AND whether they are one- or two-sided

Xnly common tests should be described solely by name; describe more complex techniques in the Methods section.

Х $\square$ A description of all covariates tested

Х $\square$ A description of any assumptions or corrections, such as tests of normality and adjustment for multiple comparisons

$\square$ A full description of the statistical parameters including central tendency (e.g. means) or other basic estimates (e.g. regression coefficient)

$\triangle \square$ AND variation (e.g. standard deviation) or associated estimates of uncertainty (e.g. confidence intervals)

For null hypothesis testing, the test statistic (e.g. $F, t, r$ ) with confidence intervals, effect sizes, degrees of freedom and $P$ value noted

Give $P$ values as exact values whenever suitable.

Х $\square$ For Bayesian analysis, information on the choice of priors and Markov chain Monte Carlo settings

$\bigotimes \square$ For hierarchical and complex designs, identification of the appropriate level for tests and full reporting of outcomes

$\triangle \square$ Estimates of effect sizes (e.g. Cohen's $d$, Pearson's $r$ ), indicating how they were calculated

Our web collection on statistics for biologists contains articles on many of the points above.

\section{Software and code}

Policy information about availability of computer code

Data collection Data was acquired using Leica LasX (version 3.5.6.21594), LAS X 4.2, Nikon Elements AR 5.2, ZEN Black SP2 and FV31 SW

Data analysis Analysis was performed using Fiji version 2.1.0/1.53c and TrackMate v6.01 plugin

For manuscripts utilizing custom algorithms or software that are central to the research but not yet described in published literature, software must be made available to editors and

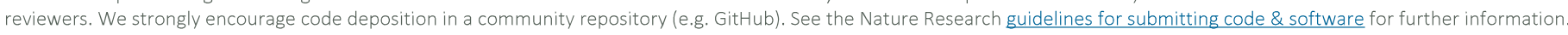

Data

Policy information about availability of data

All manuscripts must include a data availability statement. This statement should provide the following information, where applicable:

- Accession codes, unique identifiers, or web links for publicly available datasets

- A list of figures that have associated raw data

- A description of any restrictions on data availability

The raw image data used in the figures are available in OMERO/Montero Llopis et al 2021 (NatMethods) 
Please select the one below that is the best fit for your research. If you are not sure, read the appropriate sections before making your selection.

\ Life sciences

Behavioural \& social sciences

Ecological, evolutionary \& environmental sciences

For a reference copy of the document with all sections, see nature.com/documents/nr-reporting-summary-flat.pdf

\section{Life sciences study design}

All studies must disclose on these points even when the disclosure is negative.

\begin{tabular}{|c|c|}
\hline Sample size & Doesn't apply \\
\hline Data exclusions & Doesn't apply \\
\hline Replication & Doesn't apply \\
\hline Randomization & Doesn't apply \\
\hline Blinding & Doesn't apply \\
\hline
\end{tabular}

\section{Reporting for specific materials, systems and methods}

We require information from authors about some types of materials, experimental systems and methods used in many studies. Here, indicate whether each material, system or method listed is relevant to your study. If you are not sure if a list item applies to your research, read the appropriate section before selecting a response.

\begin{tabular}{|c|c|c|c|}
\hline \multicolumn{2}{|c|}{ Materials \& experimental systems } & \multicolumn{2}{|c|}{ Methods } \\
\hline $\mathrm{n} / \mathrm{a}$ & Involved in the study & $\mathrm{n} / \mathrm{a}$ & Involved in the study \\
\hline Х & $\square$ Antibodies & Х & $\square$ ChIP-seq \\
\hline Х & $\square$ Eukaryotic cell lines & Х & $\square$ Flow cytometry \\
\hline Х & $\square$ Palaeontology and archaeology & Х & $\square$ MRI-based neuroimaging \\
\hline Х & $\square$ Animals and other organisms & & \\
\hline Х & $\square$ Human research participants & & \\
\hline Х & $\square$ Clinical data & & \\
\hline Х & $\square$ Dual use research of concern & & \\
\hline
\end{tabular}

